\title{
Transcriptomic profiling of mature embryo from an elite super-hybrid rice LYP9 and its parental lines Xiaomeng $\mathrm{Ge}^{\dagger 1,2}$, Weihua Chen ${ }^{\dagger 1}$, Shuhui Song1,2, Weiwei Wang1,2, Songnian $\mathrm{Hu}^{* 1}$ and Jun $\mathrm{Yu}^{* 1}$
}

Address: ${ }^{1}$ CAS Key Laboratory of Genome Sciences and Information, Beijing Institute of Genomics, Chinese Academy of Sciences, Beijing, 100029, PR China and ${ }^{2}$ Graduate University of Chinese Academy of Sciences, Beijing, 100049, PR China

Email: Xiaomeng Ge - gexiaomeng@big.ac.cn; Weihua Chen - chen@cs.uni-duesseldorf.de; Shuhui Song - songshh@big.ac.cn; Weiwei Wang - wangweiwei@big.ac.cn; Songnian Hu* - husn@big.ac.cn; Jun Yu* - junyu@big.ac.cn

* Corresponding authors †Equal contributors

Published: II November 2008

BMC Plant Biology 2008, 8:114 doi:10.1 186/147|-2229-8-II4
Received: 20 June 2008

Accepted: II November 2008

This article is available from: http://www.biomedcentral.com/I47I-2229/8/II4

(C) $2008 \mathrm{Ge}$ et al; licensee BioMed Central Ltd.

This is an Open Access article distributed under the terms of the Creative Commons Attribution License (http://creativecommons.org/licenses/by/2.0), which permits unrestricted use, distribution, and reproduction in any medium, provided the original work is properly cited.

\begin{abstract}
Background: The mature embryo of rice (Oryza sativa, L.) is a synchronized and integrated tissue mass laying the foundation at molecular level for its growth, development, and differentiation toward a developing and ultimately a mature plant. We carried out an EST (expressed-sequencetags)-based transcriptomic study, aiming at gaining molecular insights into embryonic development of a rice hybrid triad-an elite hybrid rice LYP9 and its parental lines (93-II and PA64s)-and possible relatedness to heterosis.
\end{abstract}

Results: We generated 27,566 high-quality ESTs from cDNA libraries made from mature rice embryos. We classified these ESTs into 7,557 unigenes (2,5II contigs and 5,046 singletons) and $7,250(95.9 \%)$ of them were annotated. We noticed that the high-abundance genes in mature rice embryos belong to two major functional categories, stress-tolerance and preparation-fordevelopment, and we also identified I9I differentially-expressed genes (General Chi-squared test, $P$-value $<=0.05$ ) between LYP9 and its parental lines, representing typical expression patterns including over-dominance, high- and low-parent dominance, additivity, and under-dominance. In LYP9, the majority of embryo-associated genes were found not only abundantly and specifically enriched but also significantly up-regulated.

Conclusion: Our results suggested that massively strengthening tissue-(or stage-) characteristic functions may contribute to heterosis rather than a few simple mechanistic explanations at the individual gene level. In addition, the large collection of rice embryonic ESTs provides significant amount of data for future comparative analyses on plant development, especially for the important crops of the grass family.

\section{Background}

Heterosis is a phenomenon that particular inbred lines can produce progenies with favourable phenotypes over their parents, such as stronger tolerance to stresses and higher yields. Two fundamental hypotheses for heterosis were defined in classical genetic studies, including genome-wide dominance complementation and locusspecific over-dominant effects [1-3]. Evidence showed 
that both play roles in heterosis with involvement of epistasis [4-6]. Another theory suggested that heterosis might result from the loss of control of metabolism among heterozygotes [7]. Along with the development of genome-wide expression technology such as EST, SAGE, and microarrays, current technological advancements allow scientists to gain comprehensive information and knowledge on gene expression profiles of particular tissues from hybrids and their parental lines [8]. As it is obvious that differentially-expressed genes (DEGs) among hybrid triads may offer straightforward molecular clues to characterize phenotypic differences and genes responsible for favourable phenotypes, high-throughput gene expression profiling methods have been used for identifying DEGs in recent years. Various DEGs and their expression patterns in hybrids and their parental lines have been defined and analyzed, including over-dominance, highparent dominance, additivity, low-parent dominance, and under-dominance $[9,10]$. The distribution of DEGs varies among different samples, which not only reflects the complexity of rice transcriptomes but also points to the possibility where intricate molecular mechanisms may be involved in heterosis.

As both an important cereal crop and a model plant, the rice genome has been sequenced multiple times for its two major subspecies $[11,12]$. We have been studying rice genome with a particular interest on the molecular mechanism of heterosis as one of the specific aims proposed for the Super-hybrid Rice Genome Project (SRGP). The project focuses on an elite super-hybrid (Liang-You-Pei-Jiu, LYP9; which can yield about 20-30\% more grains; [13]) and its parental lines (93-11 and PA64s). Several datasets of DEGs as potential heterosis-associated genes as well as possible molecular mechanisms have been collected from major tissues, such as leaves, roots, and panicles $[10,14]$. The mature embryo is an important developmental stage in the rice life cycle, as it is a synchronized, undeveloped miniature plant that consists of precursor tissues for root, leaf, and stem. It was reported that its long-lived mRNA reserved in dry plant seeds might, in general, contain a valuable molecular record of embryogenic development and primary biological process [15]. A recent study suggested that the embryo of heterotic F1 possesses certain advantages at a very early developmental stage [16]. Therefore, it is important to know whether the mature embryo deposits heterotic potential for further development, as a genome-wide transcriptomic study for the mature rice embryo of a hybrid triad has not yet been reported to this date.

In this study, we constructed cDNA libraries for mature embryos from 93-11, PA64s and LYP9, and randomly sequenced approximately 10,000 ESTs from each library, resulting in 27,566 high-quality ESTs and 7,557 unigenes. We analyzed these ESTs and identified 191 DEGs between LYP9 and its parental lines (General Chi-squared test, $P$ value $<=0.05$ ). Assigning the DEGs into functional categories and expression patterns, we realized that multiple modes are at work for heterosis. All our EST sequences were submitted to the NCBI dbEST database under accession number FG943106 to FG970671.

\section{Results \\ EST acquisition}

We acquired approximately 10,000 clones from each library, which yielded 28,555 high-quality ESTs after removal of vector and low-quality sequences. The collection was further refined by removing sequences that are not anchored in the Oryza sativa Gene Index database (r17) and predicted gene sequences of the 93-11 genome [11], resulted in 27,566 high-quality ESTs that include 9,084, 9,027, and 9,455 ESTs for 93-11, PA64s, and LYP9, respectively (Table 1 ). We assembled the ESTs into 7,557 clusters, including 2,511 contigs and 5,046 singletons. The three datasets contain 3117,3938 , and 3017 unigenes from 93-11, PA64s, and LYP9, respectively, with a similar cluster-size distribution (Figure 1). Only 635 (8.4\% of total) unigenes were found universally-expressed as they are shared by all three libraries; there are about a third of them are shared by two libraries and more than $50 \%$ unigenes in each library are unique (Figure 2). Since most of the unique genes were detected as single copies, we believe that the transcriptome of a mature rice embryo is rather complex and the current sampling is not deep enough to discover low-abundance genes as most of the genes should be shared among the cDNA libraries rather than truly unique to each. Therefore, we focused our analysis on unigenes expressed in relatively high abundance.

Table I: Summary of EST sequences, contigs, and singletons in three rice cDNA libraries

\begin{tabular}{cccccc}
\hline Library ID & No. of reads & No. of high-quality EST & No. of contigs & No. of singletons & No. of unigenes \\
\hline $93-1 I$ & 10875 & 9084 & 810 & 2307 & 3117 \\
PA64s & 10751 & 9027 & 1127 & 2811 & 3938 \\
LYP9 & 11172 & 9455 & 784 & 2233 & 3017 \\
Total & 32798 & 27566 & 2511 & 5046 & 7557 \\
\hline
\end{tabular}




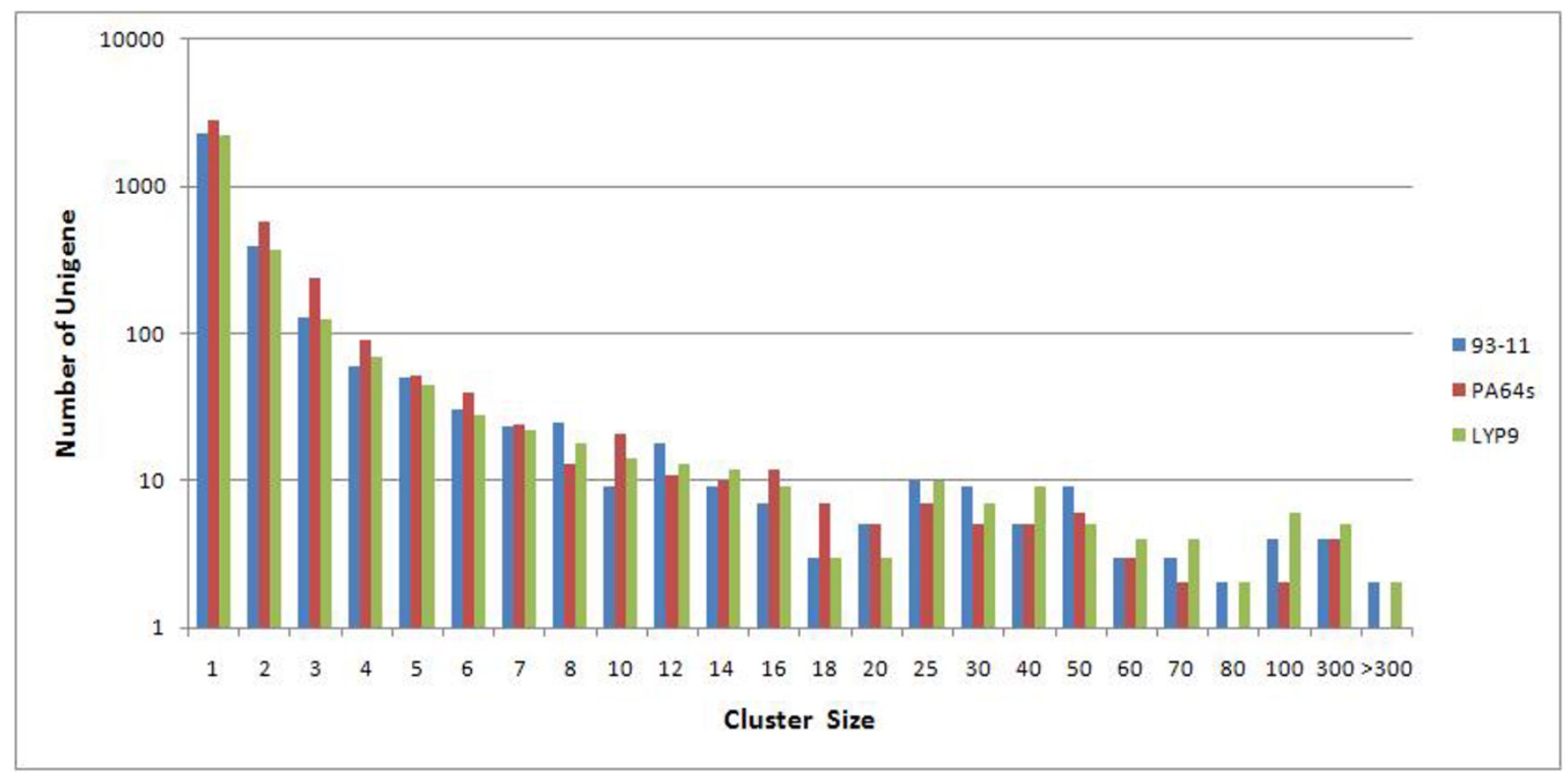

Figure I

Unigenes from three libraries sorted by cluster-size. ESTs from all libraries were clustered into unigenes (contigs and singletons) and the number of unigenes in each size-based cluster bins was plotted.

\section{Functional annotation}

We annotated 7,250 (95.9\%) out of a total of 7,557 unigenes in our dataset based on sequence similarity to those in public databases (Table 2). Among 307 un-annotated unigenes, 13 have significant similarity to the repeat sequence collection (TIGR_Oryza_Repeats.v3.3) and one to a non-coding RNA sequence from the mouse genome http://biobases.ibch.poznan.pl/ncRNA/. The remaining 293 unannotated unigenes are rather short but have open reading frames more than 30 amino acids in length, and they may represent novel protein-coding genes or noncoding RNAs as they are expressed in rice embryos.

We aligned the best hits to TIGR OGI database with the Gene Index to GO mapping database at TIGR and assigned at least one GO term to 4,565 (60.4\%) transcripts. The GO-annotated unigenes are 1,995 (64.0\%), 2,500 (63.5\%), and 1,908 (63.2\%) for 93-11, PA64s, and $L Y P 9$, respectively. We assigned most of the unigenes into several functional categories, including cellular process, metabolism process, multicellular organism development, stress tolerance, transport, cell, binding, catalytic activity, transcription regulator activity, translation regulator activity, and transporter activity (Figure 3).

We also annotated our datasets using KEGG Automatic Annotation Server http://www.genome.jp/kegg/kaas/ for pathway information (BBH method). We were able to offer 945 (12.5\%) unigenes KO numbers, including 429 $(13.8 \%), 560(14.2 \%)$, and $509(16.9 \%)$ in the libraries of 93-11, PA64s, and LYP9, respectively. The categories of metabolism, genetic information processing (GIP), environmental information processing (EIP), and cellular process involved $64.1 \%, 20.7 \%, 6.1 \%$, and $9.1 \%$ of the total annotated unigenes, respectively. As summarized in Figure 3, carbon (25.9\% of metabolism), energy (15.2\% of metabolism), and amino acid (22.3\% of metabolism) metabolisms are major contributors among the subsets of metabolism. In the category of GIP, translation ( $43.4 \%$ of GIP) and sorting (39.0\% of GIP) are the majority as opposed to transcription (11.1\% of GIP). Signal transduction $(58.1 \%$ of EIP) and membrane transport $(41.9 \%$ of EIP) constitute the majority of EIP. Other than these major categories, the rest of cellular processes showed lower abundance although defence systems (endocrine and immune systems) were found more abundant among the low-abundance categories.

\section{Expression abundance}

We calculated the expression abundance for each unigene using their cluster sizes (a sum of ESTs from all three libraries) and sorted them into three abundance levels: high-abundance ( $>=100$ ESTs), medium-abundance ( $>=$ 13 EST; this cutoff value was chosen as it represents $0.5 \%$ of the total 27,566 ESTs and is at a turning point when the number of ESTs per unigene was plotted in our redun- 


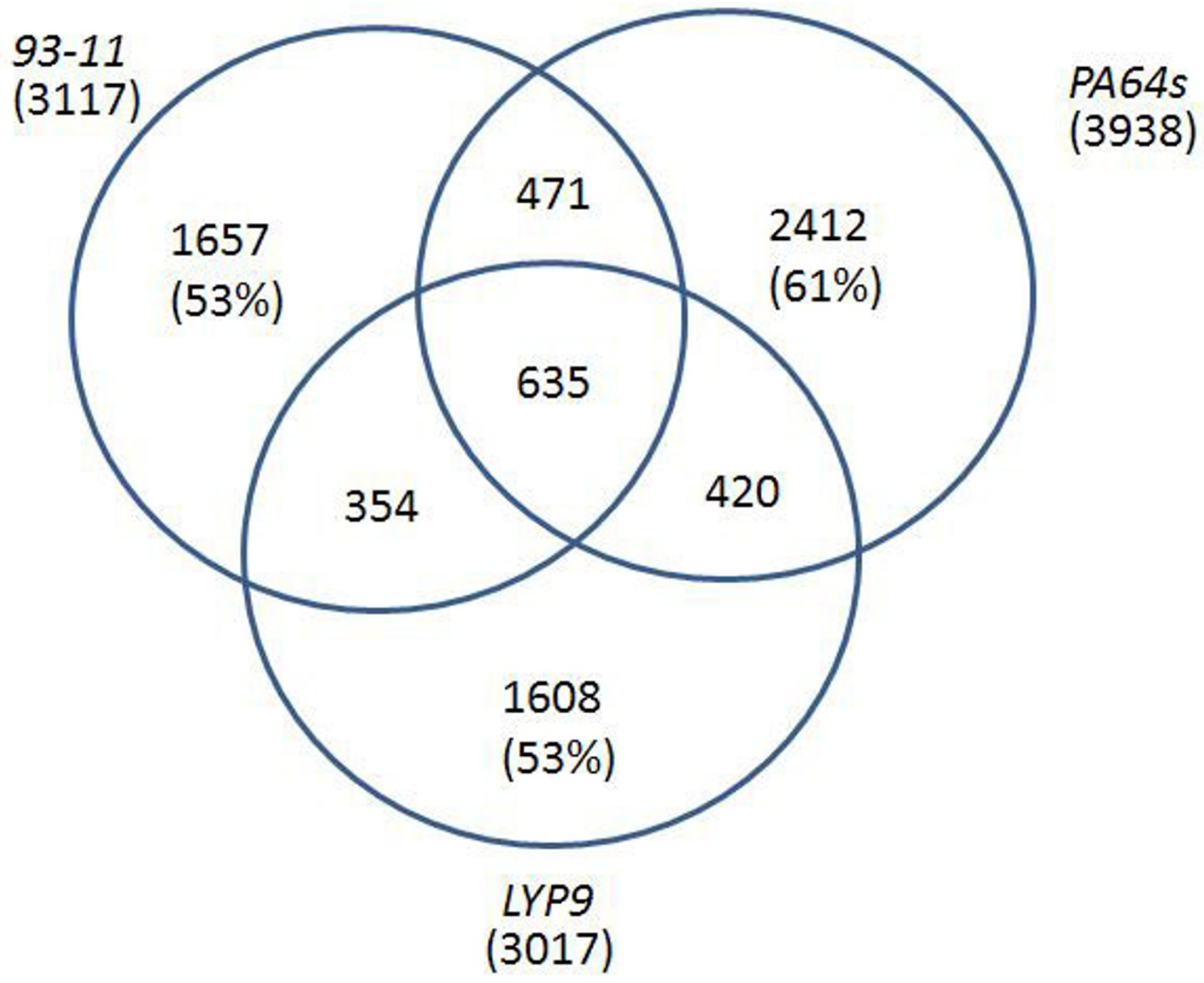

Figure 2

Venn diagrams of unigenes shared among libraries based on unigene counts.

Table 2: Annotations of unigenes based on different public databases

\begin{tabular}{lcc}
\hline \multicolumn{1}{c}{ Database } & No. of matched unigenes & Percentage of total unigenes (\%) \\
\hline TIGR OGI(verl7) & 7126 & 94.3 \\
TIGR PseudoMolecule(ver5) & 6151 & 81.4 \\
NCBI UNIGENE(ver62) & 6714 & 88.8 \\
NCBI nr protein database & 5831 & 77.2 \\
93-II BGI_Scan & 5854 & 77.5 \\
Uniprot protein database & 3628 & 48.0 \\
TIGR to GO & 4565 & 60.4 \\
KEGG Automatic Annotation Server & 945 & 12.5 \\
\hline
\end{tabular}

Alignments were performed by using blast (e-value $=1.00 \mathrm{e}-05)$, and 7,250 $(95.9 \%)$ unigenes were annotated. 
A

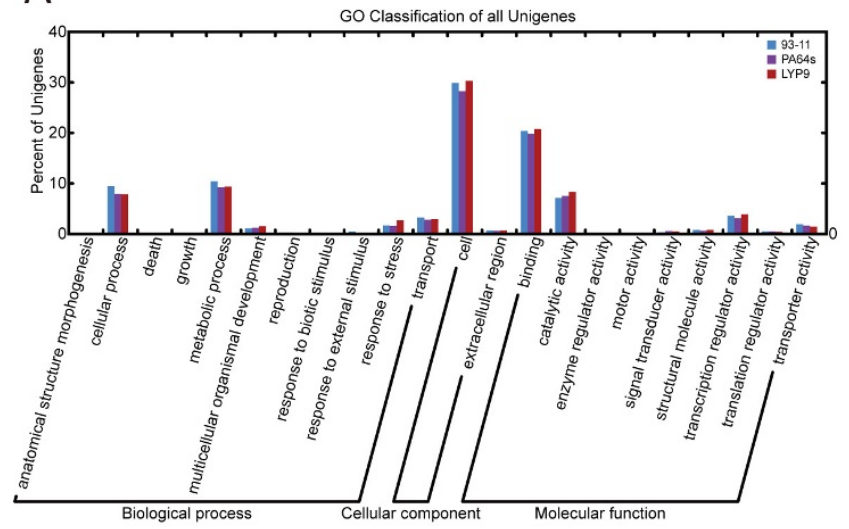

C

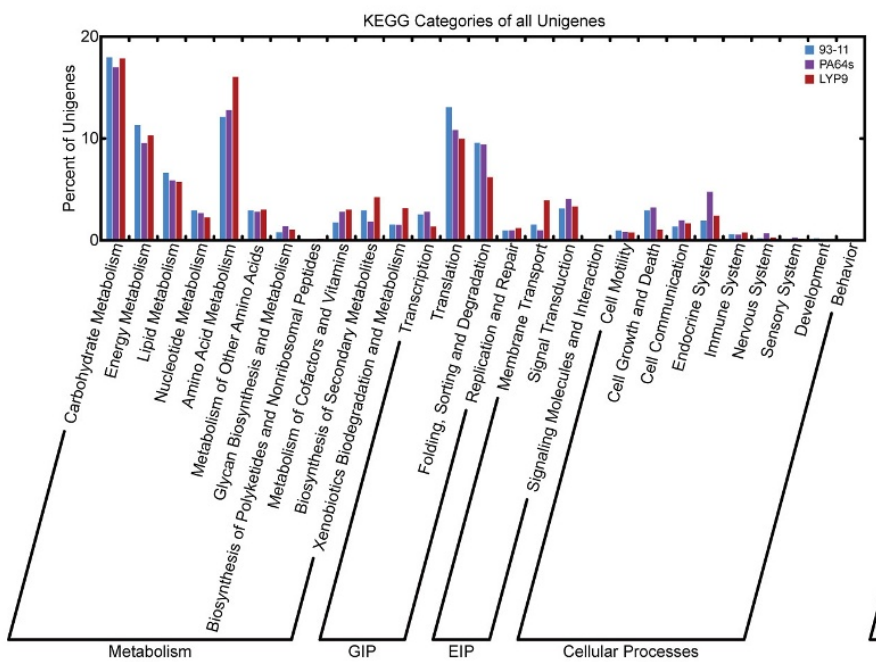

B
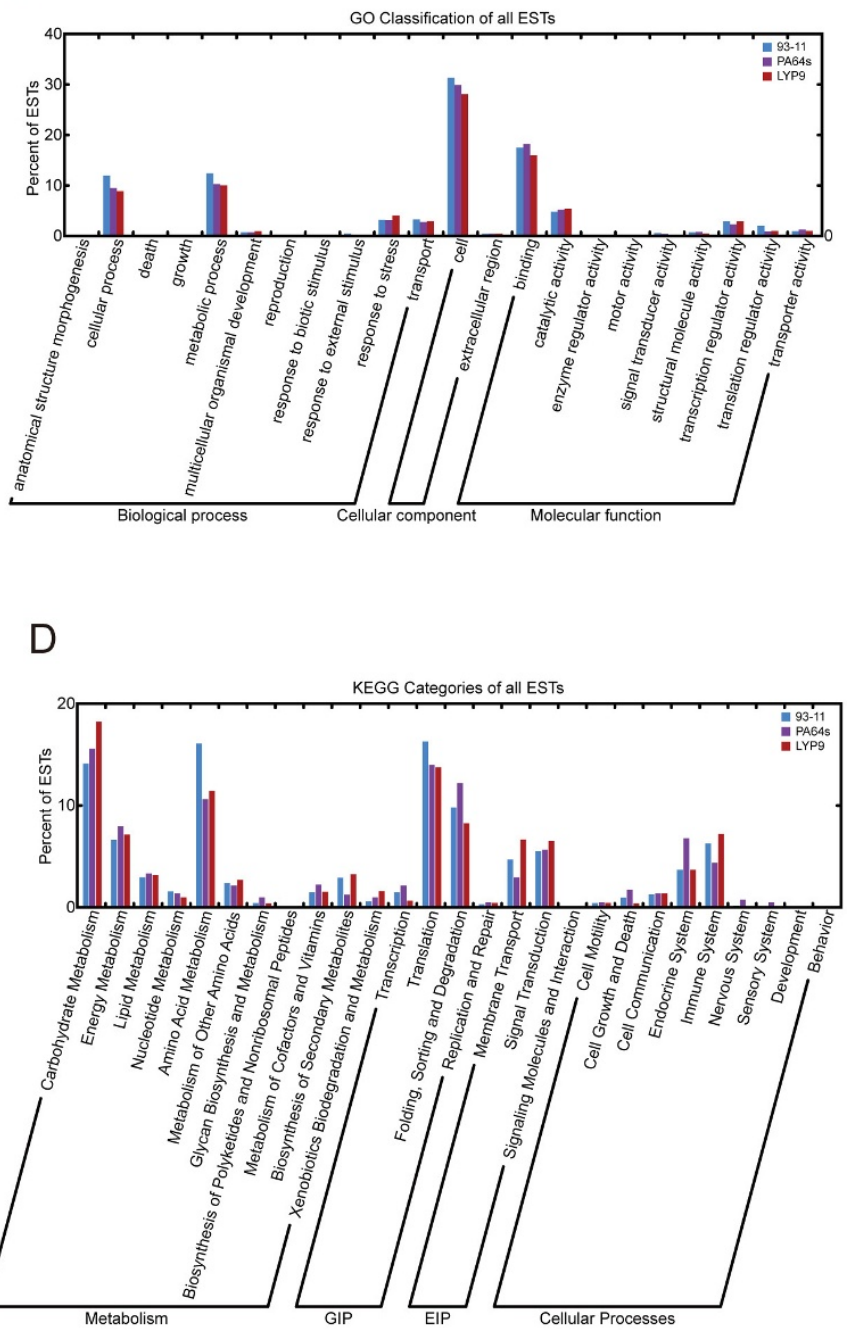

Figure 3

Functional classification of unigenes. Functional classifications are based on GO terms (A, unigenes; B, ESTs) and KEGG categories (C, unigenes; D, ESTs).

dancy analysis), and low-abundance (Table 3). The top 32 bins of high-abundance genes constitute only $0.42 \%$ of total unigenes in kind but $33.47 \%$ of the total EST counts (Table 4). The entire collection and its annotated details are listed in Additional file 1: Summary of unigenes. Their sequences are supplemented in Additional file 2: A full list of unigene sequences.

Table 3: Classification of unigenes by expression abundance

\begin{tabular}{cccc}
\hline Category & Standard & Number of genes & Number of ESTs \\
\hline High & $>=100$ & $32(0.42 \%)$ & $9227(33.47 \%)$ \\
Medium & $>=13$ & $183(2.42 \%)$ & $5315(19.28 \%)$ \\
Low & $<13$ & $7342(97.16 \%)$ & $13024(47.25 \%)$
\end{tabular}

Alignments were performed by using blast (e-value $=1.00 \mathrm{e}-05)$, and $7,250(95.9 \%)$ unigenes were annotated.
To identify embryo-characteristic genes, we carried out a hierarchical clustering analysis based on EST abundance, rice gene annotation in the unigene database at NCBI, and comparative analysis with representative libraries of other rice tissues selected from the Digital Differential Display (DDD) database (Table 5). We also did a parallel analysis utilizing SAGE data of different tissues (leaf, root, and panicle), which were generated from the same hybrid triad lines [10] based on annotations in our database that contains predicted gene sequences from the 93-11 genome. The results are rather consistent; not only is there a significant amount of high-abundance genes unique to the mature embryo (Figure 4A and $4 \mathrm{~B}$ ) but also most of the medium-abundance genes are rather characteristic for the embryo (Additional file 3: Hierarchical clustering of medium-abundance genes.). In addition, we compared 
Table 4: High-abundance genes and functional annotations

\begin{tabular}{|c|c|c|c|}
\hline Unigene ID & Abundance & TPMa & Functional annotation \\
\hline Unigene_000I & 2739 & 99354 & Cytochrome P450 monooxygenase ${ }^{b}$ \\
\hline Unigene_0002 & 1121 & 40663 & rRNA intron-encoded homing endonuclease \\
\hline Unigene_0003 & 443 & 16069 & OsIIg02II800 \\
\hline Unigene_0004 & 379 & 13748 & Em-proteinc \\
\hline Unigene_0005 & 272 & 9867 & Ramyl \\
\hline Unigene_0006 & 256 & 9286 & Lectin ${ }^{b}$ \\
\hline Unigene_0007 & 249 & 9032 & LEA3b \\
\hline Unigene_0008 & 238 & 8633 & RAB2 $I^{b}$ \\
\hline Unigene_0009 & 219 & 7944 & Thaumatin-like protein ${ }^{b}$ \\
\hline Unigene_0010 & 210 & 7618 & HSPI6.9b \\
\hline Unigene_00II & 209 & 7581 & HSP70b \\
\hline Unigene_0012 & 203 & 7364 & HSPI7.4b \\
\hline Unigene_0013 & 197 & 7146 & NAD-/NADP-dependent oxidoreductase \\
\hline Unigene_00I4 & $|7|$ & 6203 & Late embryogenesis abundant protein D-34b \\
\hline Unigene_00I5 & 164 & 5949 & Senescence-associated protein ${ }^{b}$ \\
\hline Unigene_0016 & 163 & 5913 & Glyceraldehyde-3-phosphate dehydrogenase \\
\hline Unigene_0017 & 161 & 5840 & RAB24b \\
\hline Unigene_0018 & $|5|$ & 5477 & E2 \\
\hline Unigene_0019 & 149 & 5405 & EF-I alpha \\
\hline Unigene_0020 & 144 & 5223 & Os03g0723400 \\
\hline Unigene_002I & 139 & 5042 & Rabl6bb \\
\hline Unigene_0022 & 138 & 5006 & Ec proteinc \\
\hline Unigene_0023 & 136 & 4933 & Seed maturation protein ${ }^{b}$ \\
\hline Unigene_0024 & 117 & 4244 & Hypothetical protein APECOI_2466 \\
\hline Unigene_0025 & III & 4026 & Os04g0589800 \\
\hline Unigene_0026 & 111 & 4026 & Bowman Birk trypsin inhibitorb \\
\hline Unigene_0027 & 110 & 3990 & RAB25b \\
\hline Unigene_0028 & 110 & 3990 & HSP90(8I-2)b \\
\hline Unigene_0029 & 109 & 3954 & Multiple stress-responsive zinc-finger protein ${ }^{b}$ \\
\hline Unigene_0030 & 105 & 3809 & Embryogenic-cell protein $40^{c}$ \\
\hline Unigene_0031 & 103 & 3736 & Dnal protein ${ }^{b}$ \\
\hline Unigene_0032 & 100 & 3627 & Plasma membrane associated protein \\
\hline
\end{tabular}

aTranscript per million, ${ }^{\mathrm{b}}$ stress-tolerance associated genes, and c development-associated genes.

embryo-associated genes against all predicted genes from the parental genomes (93-11 and PA64s) based on GO functional classification, and observed the enrichment of genes in several categories, such as response to stimulus and stress in the category Biological Processes (Figure 4C).

Based on GO functional classification, we found that (1) high-abundance genes are enriched in cellular and meta- bolic processes, response to stress, cell, binding, catalytic activity, and translation regulator activity; (2) mediumabundance genes are those for embryo development, response to external stimulus, transport, enzyme regulator activity, signal transducer activity, structural molecule activity, transcription regulator activity, and transporter activity; and (3) low-abundance genes are involved in anatomical structure morphogenesis, death, growth,

Table 5: Libraries for Digital-Differential-Display analysis

\begin{tabular}{|c|c|c|c|c|}
\hline Tissue & Lib ID & EST & Clustered EST & Unigene \\
\hline Callus & 19058 & 53637 & 50361 & 10713 \\
\hline Flower & 19057 & 46187 & 43264 & 10451 \\
\hline 9 leaf-stage leaf & 19096 & 13230 & $1289 \mid$ & 681 \\
\hline Drought stress (leaf) & 9652 & 5615 & 4154 & 1344 \\
\hline Panicle & 19050 & $|357|$ & 13060 & 3634 \\
\hline Root of seedlings & 19053 & 17250 & 16565 & 4445 \\
\hline Seed & 19082 & 9792 & 9408 & 4228 \\
\hline Mature rice embryo & local data & 28555 & 27566 & 7557 \\
\hline
\end{tabular}

Data from this study are highlighted in bold and italic. 
A
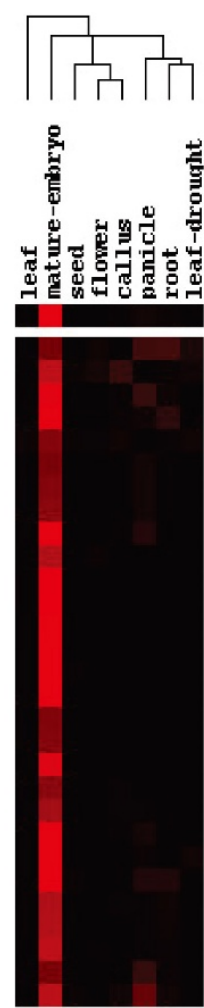

Os. 12366

$0 s .57475$

Os. 12244

Ds. 12241

$0 s .28384$

0 . 5717

$0 s .3419$

0 s. 6618

$0 s .54304$

0s. 78916

Os. 6394

0s. 6394

$0 s .36328$

Ds. 22571

0s. 51718

Ds. 83904

$0 s .22404$

$0 s .37616$

$0 s .13800$

$0 s .74608$

Os.46051

s. 12812

$0 s .27956$

Os. 12551

0s.12080

0s. 54997

Os. 5499

Os. 7862

$0 s .48741$
B
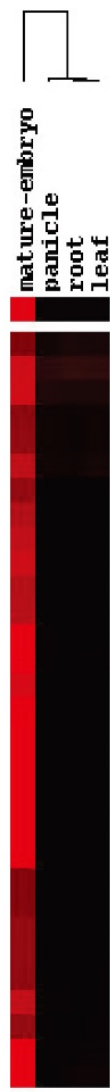

9311 Chr03 0387

9311 Chr02 2530

9311 Chro3 1065

9311 Chr04 1968

9311 Chr09 1478

9311 Chro3 0613

9311 Chr05 1048

9311 Chr03 0477

9311 Chr03 3587

9311 Chr10 1955

9311 rhr11 1171

9311 Chro3 1229

9311 Chr11 2276

9311 Chr06 1547

9311 Chr05 0314

9311 Chr01 0276

9311 Chr04 0525

9311 chr 04 a

9311 Chr04 2862

9311 Chr05 2724

9311 Chro5 1464

9311 Scaffoldo12143_1

9311 Chr04 2117

9311 Chro7 1210

9311 Chr01 3416

9311 Chr04 2652

9311 Chu 0401

9311 Chr06 2401

931 Chr06 0708

9311 Chro1 0194

9311 Chr03 1081

9311 Chr11 1172

C

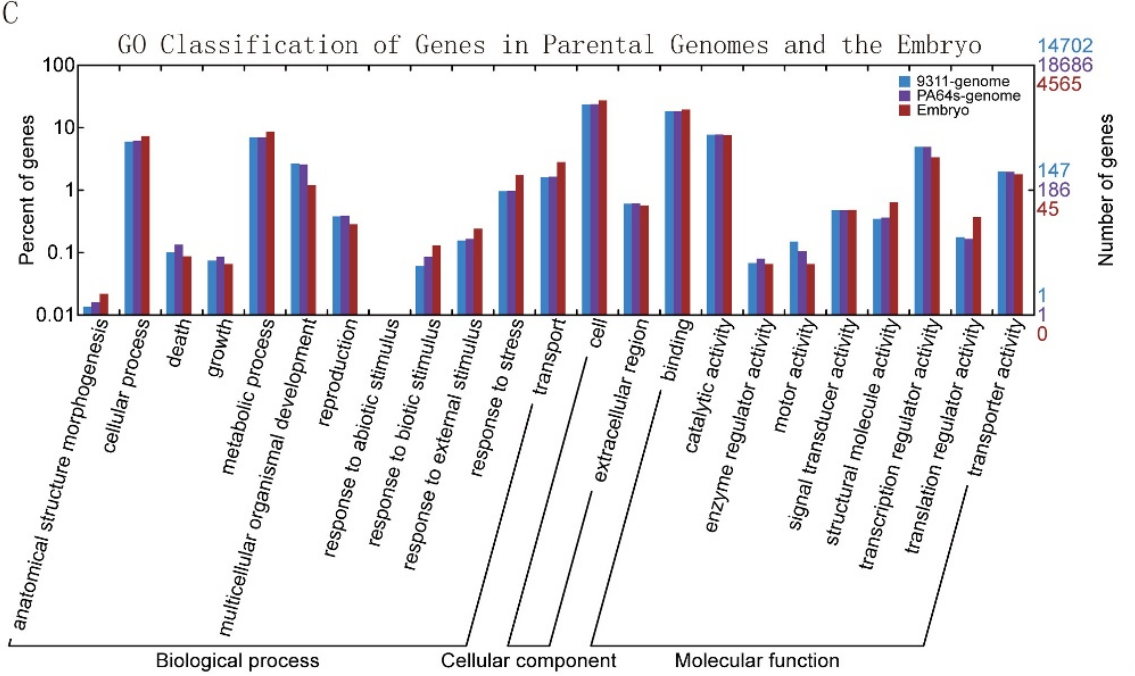

Figure 4

Hierarchical clustering and $\mathbf{G O}$ analyses of embryo-associated genes. We summarized our hierarchical clustering analyses (A, compared to NCBI DDD database; B, compared to SAGE data generated from the same hybrid line) of high-abundance genes based on data from the embryo and other tissues (also see Table 5). The expression abundance was normalized to transcript per million. The frequencies of unigenes from different libraries are indicated by color intensity (red), and blank was adjusted to be black. A GO classification of embryo-associated genes in comparison to the genome total (C) was also showed to emphasize the fact that the enrichment of stress- and development-associated genes in the mature embryo. 
A

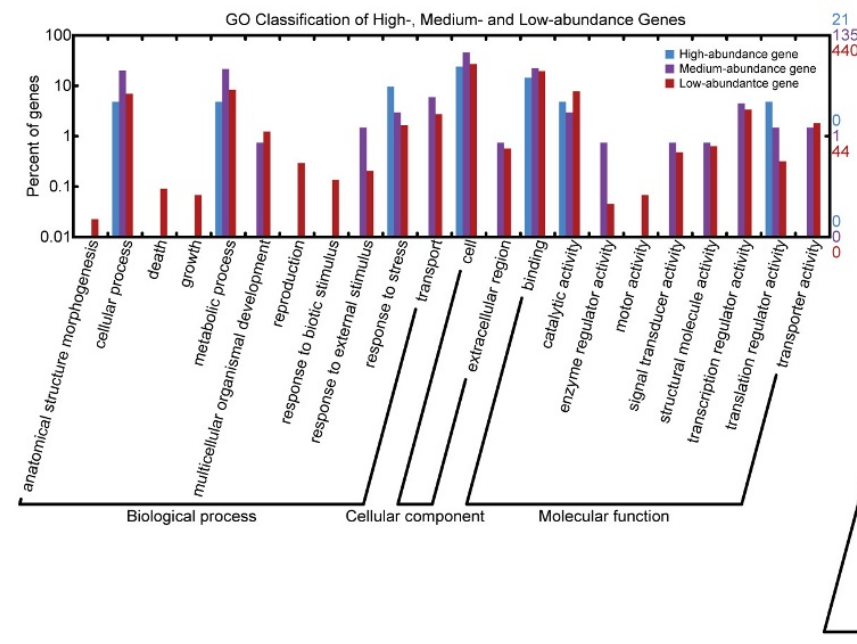

B

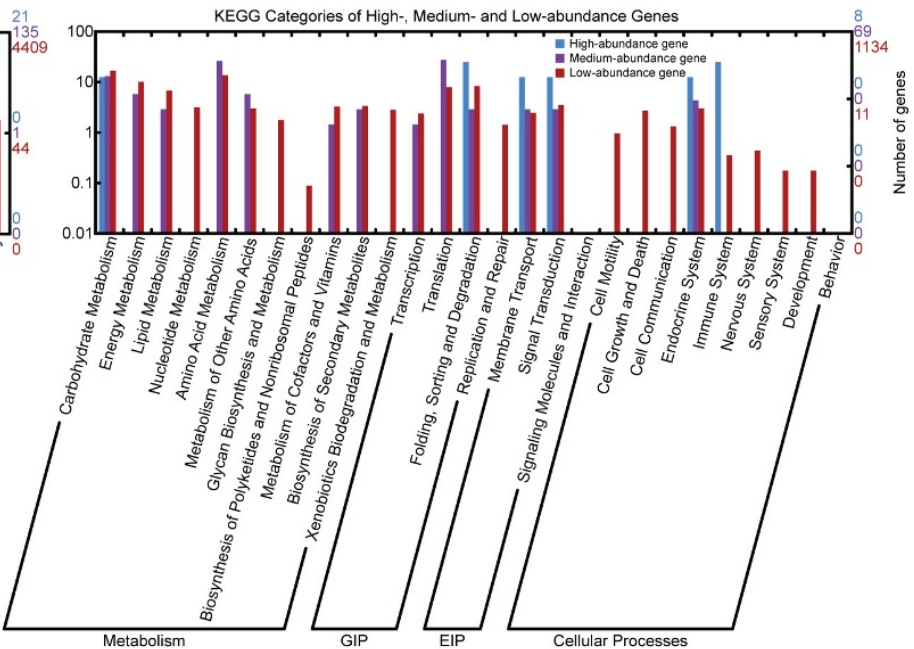

Figure 5

Functional classification by expression abundance. GO classification and KEGG pathway analyses based on gene expression abundance. GO classes (A) and KEGG pathways (B) of all unigenes sorted by expression abundance described in Table 3 were shown. The high-abundance genes represent the most important functional categories and pathways in the mature embryo.

reproduction, response to biotic stimulus, and motor activity (Figure 5).

We also analyzed the genes classified as different pathways. We found that (1) high-abundance genes concentrate on carbohydrate metabolism, folding/sorting/ degradation, membrane transport, signal transduction, and defence (endocrine and immune) systems, and (2) medium-abundance genes are mainly involved in the pathways of energy metabolism, lipid metabolism, amino acid metabolism, transcription and translation (Figure 5). High-abundance genes were found in the pathways of glycolysis, pores ion channels, protein folding and associated processing, ubiquitin-mediated proteolysis, MAPK signalling, progesterone-mediated oocyte maturation, and antigen processing/presentation; all appear performing important functions in keeping embryonic status and rapid growth recovery.

\section{Abundant embryo-characteristic gene families}

We discovered four major gene families expressed in highabundance in the rice embryo (Table 6). The most abundant gene family belongs to cytochrome P450 monooxygenases, making up $10 \%$ of total ESTs. They are universally-expressed and senescence-associated in all eukaryotic species. Among plants, these enzymes are important for the biosynthesis of hormones, defensive compounds, and fatty acids. The second group is composed of HSPs (heat shock proteins), and they are multifunctional molecular chaperones and many show over- expression during embryonic development and are inducible by desiccation and other stress types. We identified four low-molecular-weight (LMW, 17-30 KD) HSPs in our dataset; they are HSP16.9, HSP17.4, HSP22, and HSP26 as well as four classical HSPs: HSP40, HSP70, HSP90, and HSP101. In numbers, there are $16 \%$ of the abundantly-expressed genes and 3.3\% of the total ESTs belonging to the HSP family. The third protein family is the LEA (late embryogenesis abundant; $3.0 \%$ of the total ESTs) proteins that are a group of proteins accumulating in high abundance during last stages of seed formation and periods of the water shortage in vegetative organs. Ample evidence bsuggested that LEA proteins, especially its subgroup 3 members, are involved in desiccation resistance through a variety of machineries, including water retention, ion sequestration, and direct protein protection $[17,18]$. The fourth group is the RAB family, which are membrane-associated small GTP-binding proteins localized to discrete subcellular compartments and involved in signal transduction, cytoskeleton organization, and vesicle trafficking. They are also stress-inducible. We identified its four members whose ESTs make up 2.6\% of the total collection: RAB16 (b, c, d), RAB21, RAB24, and RAB25.

\section{DEGs between LYP9 and its parents}

Based on P-values of 0.05 and 0.01 from general Chisquared test, we defined $191(2.53 \%)$ and 89 (1.12\%) DEGs, respectively, between LYP9 and its parental lines (Table 7). The full list of all DEGs is in Additional file 4: 
Table 6: Abundant gene families detected in the dataset

\begin{tabular}{|c|c|c|c|c|c|c|c|}
\hline \multirow[t]{2}{*}{ Gene family } & \multicolumn{2}{|l|}{ Subfamily } & \multicolumn{4}{|c|}{ Expression abundance } & \multirow[t]{2}{*}{ Unigene ID } \\
\hline & & & $93-11$ & PA64s & LYPQ & Total & \\
\hline $\mathrm{P} 450^{\mathrm{a}}$ & \multicolumn{2}{|c|}{ Cytochrome P450 Monooxygenase } & IIII & 348 & 1280 & 2739 & Unigene_000I \\
\hline \multirow[t]{8}{*}{$\mathrm{HSPb}$} & LMW & Hspl6.9 & 29 & 102 & 79 & 210 & Unigene_0010 \\
\hline & & Hspl7.4 & 28 & 74 & 101 & 203 & Unigene_0012 \\
\hline & & Hsp22 & 3 & 3 & 13 & 19 & Unigene_0I4I \\
\hline & & Hsp26 & 4 & 6 & 40 & 50 & Unigene_0055 \\
\hline & Classical HSP protein & Hsp40 & 29 & 45 & 29 & 103 & Unigene_003I \\
\hline & & Hsp70 & 71 & 50 & 88 & 209 & Unigene_00II \\
\hline & & Hsp90 & 44 & 34 & 32 & 110 & Unigene_0027 \\
\hline & & Hspl0I & 1 & 4 & II & 16 & Unigene_0I70 \\
\hline \multirow[t]{13}{*}{ LEAc } & LEA & LEA3 & 90 & 61 & 98 & 249 & Unigene_0007 \\
\hline & & LEA D-34 & 80 & 16 & 75 & 171 & Unigene_0014 \\
\hline & & LEA & 21 & 7 & 27 & 55 & Unigene_0052 \\
\hline & & LEA & 8 & 17 & 13 & 38 & Unigene_0072 \\
\hline & & LEA & 12 & 3 & 19 & 34 & Unigene_008I \\
\hline & Seed maturation protein & PM & 61 & 19 & 56 & 136 & Unigene_0023 \\
\hline & & PM24 & 8 & 6 & 9 & 23 & Unigene_0I23 \\
\hline & & PM & 4 & 2 & 11 & 17 & Unigene_0I5I \\
\hline & & PM & 4 & 4 & 7 & 15 & Unigene_0180 \\
\hline & & PM & 3 & 5 & 4 & 12 & Unigene_0228 \\
\hline & & PM & 3 & I & 0 & 4 & Unigene_0848 \\
\hline & & PM23 & 1 & 1 & I & 3 & Unigene_0999 \\
\hline & & PM36 & 2 & 1 & 0 & 3 & Unigene_1204 \\
\hline \multirow[t]{6}{*}{$\mathrm{RAB}^{\mathrm{d}}$} & RAB2I & & 85 & 52 & 101 & 238 & Unigene_0008 \\
\hline & RAB24 & & 64 & 29 & 68 & 161 & Unigene_0017 \\
\hline & RABI6 & RABI6b & 46 & 27 & 66 & 139 & Unigene_002I \\
\hline & & RABI6c & 27 & 11 & 26 & 64 & Unigene_0047 \\
\hline & & RABI6d & 1 & 4 & 6 & II & Unigene_0247 \\
\hline & RAB25 & & 45 & 13 & 52 & 110 & Unigene_0028 \\
\hline
\end{tabular}

aCytochrome P450 family, bheat shock protein, clate embryogenesis abundant protein, and d RAB protein.

DEGs and their expression patterns. Most of these DEGs belong to high- and medium-abundance genes, $84.3 \%$ and $59.2 \%$, respectively. We focus our discussion on the larger group (P-value 0.05) unless specified. The DEGs cover almost all expression patterns with rather unequal distributions. First, the majority of DEGs $(84.3 \%, 161)$ exhibit either high- or low-parent dominances rather than deviate significantly from both $(15.7 \%, 30)$. Second, of the 161 genes, $72.7 \%$ (117) show differences between PA64s and LYP9, whereas only 27.3\% (44) exhibit differences between 93-11 and LYP9. This result indicated that the gene expression pattern of LYP9 is more similar to 9311 than to PA64s at this developmental stage. Third, there are slightly less high-parent dominant genes (76) than low-parent dominant ones (85) in embryo both between 93-11 and LYP9 (18/26) and between PA64s and LYP9 (58/59), whereas over-dominant genes (20) are more than under-dominant genes (6). Finally, there are slightly more up-regulated genes than down-regulated genes in general. When we used the more stringent P-value (0.01), most of the observed rules did not change except that the up-regulated genes between PA64s and LYP9 were found slightly more than down-regulated genes (36/28). In general, the distribution pattern of DEGs in the embryo is similar to that of the panicle as compared to our previous SAGE study [10]. When we displayed the fold change of DEGs $(L Y P 9 /[(93-11+P A 64 s) / 2])$ in a two-dimensional plot (Figure 6), the consistency remains.

\section{Integrated analysis with proteomic and SAGE data}

We compared our DEGs to those identified from proteomic data generated from the same material (Weiwei Wang, personal communication). The proteomic data were acquired from the same material as what we used in this study, mature embryo of 93-11, PA64s, and LYP9. Over 1,300 2-D gel spots (putative proteins) were ana- 
Table 7: Differentially-expressed genes between FI hybrid (LYP9) and its parental lines (93-II and PA64s)

\begin{tabular}{|c|c|c|c|c|c|c|}
\hline \multirow[t]{3}{*}{ Genetic class } & \multirow[t]{3}{*}{ Subclass } & \multirow[t]{3}{*}{ Expression pattern } & \multicolumn{4}{|c|}{ P-value(total number of DEGs) } \\
\hline & & & \multicolumn{2}{|c|}{$0.05(191)$} & \multicolumn{2}{|c|}{$0.01(89)$} \\
\hline & & & \# & $\%$ & $\#$ & $\%$ \\
\hline \multirow[t]{5}{*}{ Up-regulated } & Over-dominance & $\mathrm{L}>\mathrm{N}>\mathrm{P}$ & 5 & 2.6 & 3 & 3.4 \\
\hline & & $\mathrm{L}>\mathrm{P}>\mathrm{N}$ & 0 & 0 & 0 & 0 \\
\hline & & $L>N=P$ & 15 & 7.9 & 8 & 9 \\
\hline & High-parent dominance & $L=P>N$ & 18 & 9.4 & 6 & 6.7 \\
\hline & & $L=N>P$ & 58 & 30.4 & 36 & 40.4 \\
\hline \multirow[t]{2}{*}{ Additivity } & Additivity & $\mathrm{N}<\mathrm{L}<\mathrm{P}$ & 1 & 0.5 & 0 & 0 \\
\hline & & $\mathrm{P}<\mathrm{L}<\mathrm{N}$ & 3 & 1.6 & 0 & 0 \\
\hline \multirow[t]{5}{*}{ Down-regulated } & Low-parent dominance & $\mathrm{L}=\mathrm{P}<\mathrm{N}$ & 26 & 13.6 & 7 & 7.9 \\
\hline & & $L=N<P$ & 59 & 30.9 & 28 & 31.5 \\
\hline & Under-dominance & $\mathrm{L}<\mathrm{N}<\mathrm{P}$ & 2 & 1 & 0 & 0 \\
\hline & & $\mathrm{L}<\mathrm{P}<\mathrm{N}$ & 0 & 0 & 0 & 0 \\
\hline & & $\mathrm{L}<\mathrm{P}=\mathrm{N}$ & 4 & 2.1 & 1 & I.I \\
\hline
\end{tabular}

$\mathrm{N}, \mathrm{P}$, and $\mathrm{L}$ stand for paternal lines (93-II), maternal lines (PA64s), and FI hybrids (LYP9), respectively.

lyzed and 54 differentially-expressed proteins were identified in the study. We found that most of the highabundance genes also highly expressed in the proteomic data, but the expression patterns were not always consistent. There were only nine genes found consistent in both datasets, and three of them were not (Table 8, when there are several protein spots for one gene and one of the spots are consistent with the EST data, we classified this gene as consistent). Seven of the consistent DEGs were found expressed higher in LYP9 except early embryogenesis protein and glutelin; both are down-regulated. Most of the DEGs in the protein data fell into three general categories: additivity, over-dominance, and under-dominance, whereas most of the DEGs generated from the EST data are in other groups: high- and low-parent dominance. We also compared our EST data to those of our SAGE experiments generated from the same hybrid triad. The SAGE data were generated from nine SAGE libraries made from three tissues: root, leaf, and panicle of the same hybrid triad, 93-11, PA64s, and LYP9. Roughly ten-thousand SAGE tags from each library were obtained and 20,595 unique tags were annotated, and among them, 1,216 DEGs $(\mathrm{p}<0.01)$ were detected [19]. In this study, we identified 22 DEGs between the two datasets, which are shared by at least one tissue (library). The expression patterns in both dataset were summarized in Additional file 5: DEGs shared with SAGE data. These genes are more likely to be associated with heterosis, especially when some of the DEGs have consistent expression pattern in all tissues.

\section{Alignments of DEGs to genome sequences}

To understand the regulatory mechanism of DEGs, we traced their genomic sequences in 93-11 and PA64s, and identified the regulatory and transcript sequences among orthologous gene pairs. We scrutinized the genomic sequences $(3 \mathrm{~kb}$ upstream from the translational start codon including 5'UTR, $3 \mathrm{~kb}$ downstream from the translational stop codon including 3'UTR, and coding region) from the genome sequences of 93-11 and PA64s for the 22 DEGs that are confirmed with our SAGE data. We found that $9(41 \%)$ genes have almost identical sequences in all regions (without deletion/insertions more than $3 \mathrm{bp}$ in length), and we believed that these genes might be regulated by distant trans-regulatory mechanisms other than cis-elements in their immediate promoters. Among the 13 (59\%) genes that showed significant sequence deviations, $5(23 \%), 10(45 \%)$, and $11(50 \%)$ genes have differences in coding, 5'UTR, and 3' UTR regions, respectively. We also analyzed well-defined regulatory sequence motifs and found that 14 (64\%) genes have obvious motif variations. Our results were summarized in Table 9.

Quantitative PCR-based Experimental validation of DEGs We chose eight stress-tolerance-associated and four development-associated genes-both expressed in high-abundance and functionally important to mature rice embryoto validate their expression patterns, using quantitative real-time PCR or qRT-PCR. The stress-tolerance-associated proteins are LEA3, HSP17.4, HSP70, tonoplast intrinsic protein, ubiquitin E2, thaumatin-like protein, BowmanBirk trypsin inhibitor, and cold-regulated protein (gi| $115464405 \mid$ ). The four development-associated genes included one embryo-specific protein and three metabolism-associated proteins (succinate dehydrogenase, NADP-dependent oxidoreductase and S-adenosylmethionine decarboxylase). Almost all the results showed consistent trends with their corresponding EST data (Figure 

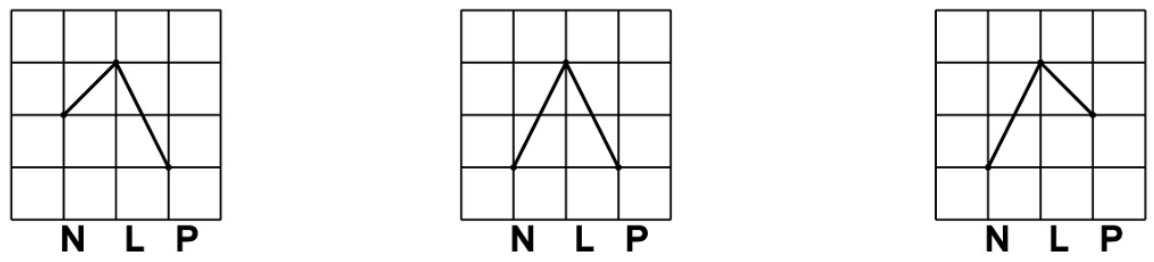

\section{Mature embryo}
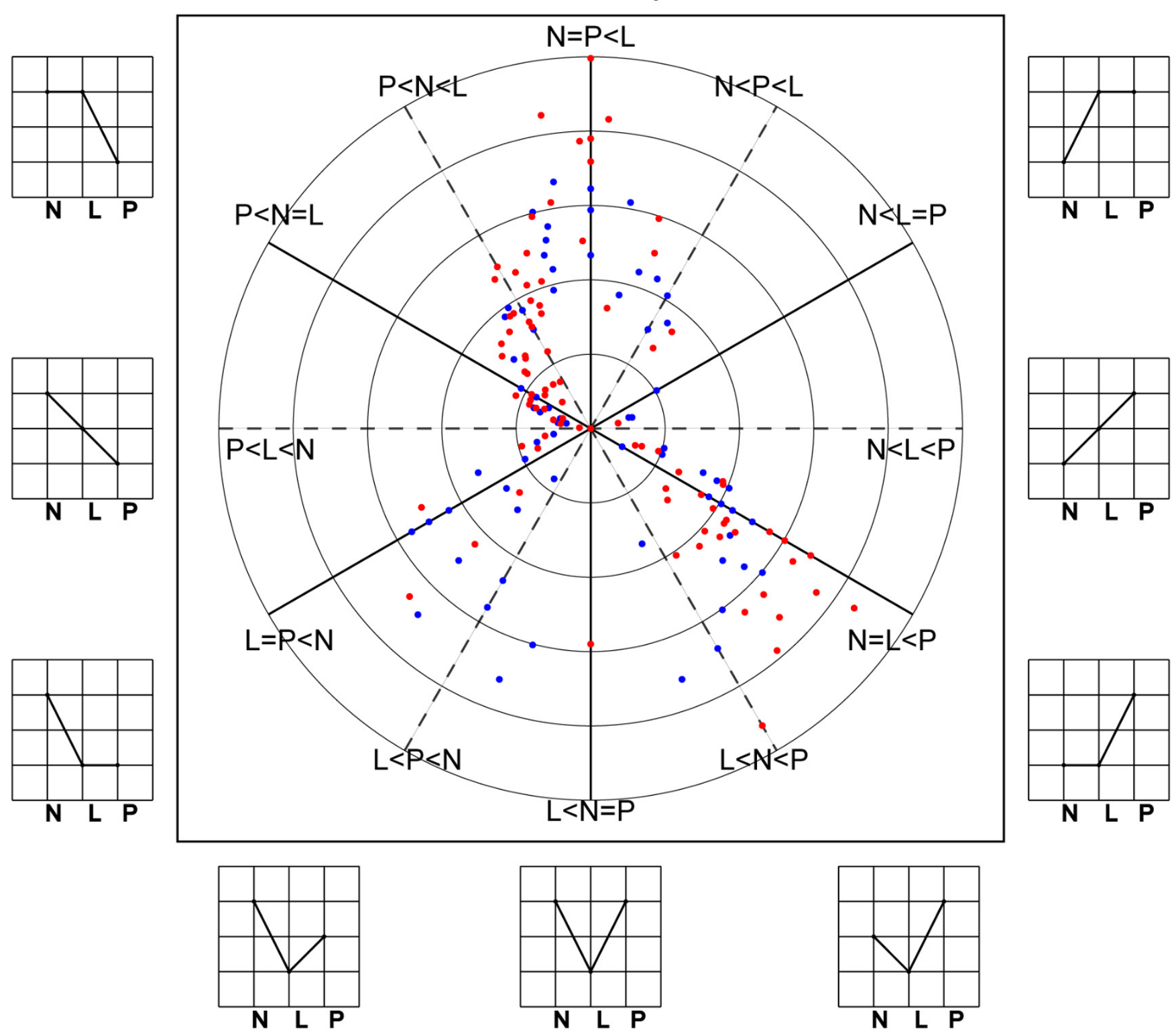

Figure 6

Distribution of DEGs by fold changes. A two-dimensional display of expression profiling and fold changes of DEGs in mature rice embryos. N, L, and $\mathrm{P}$ stand for 93-II, LYP9, and PA64S, respectively. 12 different expression projections are displayed clock-wise according to their fold changes. The radius at which a gene is plotted represents fold changes $L /[(P+N) / 2]$. Spots falling on the horizontal and vertical lines exhibit pure additivity and over- (or under-) dominance, respectively. 
Table 8: DEGs shared by EST and protein data

\begin{tabular}{|c|c|c|c|c|c|c|}
\hline \multirow[t]{2}{*}{ Unigene ID (description) } & \multicolumn{3}{|c|}{ EST(copy number) } & \multicolumn{3}{|c|}{ Protein (vol\%) } \\
\hline & $93-11$ & PA64s & LYP9 & $93-11$ & PA64s & LYPQ \\
\hline \multicolumn{7}{|l|}{ Consistent } \\
\hline Unigene_0007 (LEA3) & 90 & 61 & 98 & 0.962 & - & 0.326 \\
\hline Unigene_0012 (sHSP) & 28 & 74 & 101 & 0.323 & 0.721 & 0.782 \\
\hline \multirow[t]{3}{*}{ Unigene_0025 (Bowman Birk trypsin inhibitor) } & 49 & 13 & 49 & 0.413 & 0.571 & 0.856 \\
\hline & & & & 2.64 & 0.643 & 0.808 \\
\hline & & & & 0.835 & 0.217 & 0.203 \\
\hline \multirow[t]{3}{*}{ Unigene_0054 (Lipoprotein) } & 12 & 4 & 35 & 0.896 & 1.18 & 2.33 \\
\hline & & & & 0.321 & 0.741 & - \\
\hline & & & & 0.462 & 1.14 & 0.751 \\
\hline \multirow[t]{2}{*}{ Unigene_0006 (Lectin) } & 106 & 53 & 97 & 0.952 & 0.544 & 0.211 \\
\hline & & & & 0.121 & 0.158 & 0.405 \\
\hline Unigene_0299 (Enolase) & 0 & 5 & 4 & 0.421 & 0.595 & 0.736 \\
\hline Unigene_0048 (Embryo-specific protein) & 21 & 9 & 33 & 0.624 & 0.883 & 1.67 \\
\hline \multirow[t]{3}{*}{ Unigene_0073 (Early embryogenesis protein) } & 26 & 3 & 9 & 0.145 & 0.136 & 0.298 \\
\hline & & & & 0.286 & - & 0.197 \\
\hline & & & & 0.392 & 0.067 & 0.073 \\
\hline \multirow[t]{3}{*}{ Unigene_0442 (Glutelin) } & 1 & 6 & 0 & 0.154 & 0.319 & 0.824 \\
\hline & & & & 0.202 & 0.732 & 0.261 \\
\hline & & & & 0.242 & 0.38 & - \\
\hline \multicolumn{7}{|l|}{ Not Consistent } \\
\hline \multirow[t]{2}{*}{ Unigene_0093 (PreproMP73) } & 8 & 5 & 17 & 0.184 & 0.146 & - \\
\hline & & & & 0.061 & 0.303 & 0.104 \\
\hline Unigene_00II (HSP70) & 71 & 50 & 88 & 0.421 & 0.923 & 0.332 \\
\hline Unigene_0253 (Superoxide dismutase) & I & 8 & 2 & 0.434 & - & 0.301 \\
\hline
\end{tabular}

7). We listed the primer sequences used in the experiment in Additional file 6: Primers for qRT-PCR.

\section{Discussion}

Genes in abundance: stress tolerance and development

High-abundance genes are known to characterize tissueor organ-specificity since they are dominant in manifestation of major functions of the tissue or organ. We found that the embryo shares 3,649,3,670, and 2,768 genes with leaf, panicle, and root, respectively, and there are 2,342 genes common to all tissues. All high-abundance and most medium-abundance genes in our dataset are either specifically expressed or unique to their tissues of origin whereas only 47 genes in medium-abundance expressed universally at similar expression level, as compared to the SAGE data, distributing among the most basic GO categories (Additional file 7: GO classification of universally-expressed genes among embryo and other tissues from SAGE data.). In an attempt to define functional characteristics of the mature rice embryo based on its high- and medium-abundance genes, we found that two major functional categories stood out: stress tolerance and development, which are composed of $56.3 \%$ and
$9.4 \%$ of high-abundance genes, respectively. Furthermore, there are still large amount of genes in the universally expressed categories such as metabolism and cellular process which expressed specifically in high-abundance in mature embryo.

We identified a large number of genes that are in the category of anti-stress via various mechanisms, which include a complete water-deficit tolerance system and genes involved in resistance in oxidation damage and fungal pathogen infection (Table 6 and Table 10). Water deficit is a major threatening factor to embryos, and a water-deficit tolerance system in plants was reported, including membrane proteins, chaperones (mainly HSPs and LEAs), water channel proteins, protease inhibitors, proteases, and the ubiqutin system [17]. The three high-abundance gene families (LEA, HSP and RAB) that we categorized in this study all have important functions in this system $[17,18]$. Our proteomic study with the same material also provided a footnote to this conclusion (Weiwei Wang, personal communication). 
Table 9: DEGs in parental genomes

\begin{tabular}{|c|c|c|c|c|c|}
\hline $93-11$ & PA64s & 5'UTR & Coding & 3'UTR & Motif \\
\hline 93II_Chr0I_0275 & Pa64_Chr0I_0330 & $\mathrm{N}$ & $\mathrm{N}$ & $\mathrm{N}$ & $Y$ \\
\hline 931I_Chr02_0049 & Pa64_Chr02_0052 & $\mathrm{N}$ & $\mathrm{N}$ & $\mathrm{N}$ & $\mathrm{N}$ \\
\hline 931I_Chr02_2243 & Pa64_Chr02_2903 & $\mathrm{N}$ & $\mathrm{N}$ & $\mathrm{N}$ & $\mathrm{N}$ \\
\hline 931I_Chr03_0335 & Pa64_Chr03_0342 & $\mathrm{N}$ & $\mathrm{N}$ & $\mathrm{N}$ & $\mathrm{N}$ \\
\hline 931I_Chr03_I553 & Pa64_Chr02_I74I & $\mathrm{N}$ & $\mathrm{N}$ & $\mathrm{N}$ & $\mathrm{N}$ \\
\hline 931I_Chr03_2170 & Pa64_Chr02_I375 & $\mathrm{N}$ & $\mathrm{N}$ & $\mathrm{N}$ & $\mathrm{N}$ \\
\hline 931I_Chr05_2916 & Pa64_Chr05_2748 & $\mathrm{N}$ & $\mathrm{N}$ & $\mathrm{N}$ & $\mathrm{N}$ \\
\hline 93II_Chr05_2972 & Pa64_Chr05_2805 & $\mathrm{N}$ & $\mathrm{N}$ & $\mathrm{N}$ & $\mathrm{N}$ \\
\hline 931I_Chr07_I7I4 & Pa64_Chr07_1619 & $\mathrm{N}$ & $\mathrm{N}$ & $\mathrm{N}$ & $\mathrm{N}$ \\
\hline 931I_Chr0I_4347 & Pa64_Chr0I_4085 & $\mathrm{N}$ & $\mathrm{N}$ & $Y$ & $Y$ \\
\hline 93II_Chr03_0234 & Pa64_Chr03_024I & $\mathrm{N}$ & $\mathrm{N}$ & $Y$ & Y \\
\hline 931I_Chr10_1957 & Pa64_Chrlo_1617 & $\mathrm{N}$ & $\mathrm{N}$ & $Y$ & $Y$ \\
\hline 93II_Chr0I_2I24 & Pa64_Chr0I_2I74 & $Y$ & $N$ & $\mathrm{~N}$ & $Y$ \\
\hline 93II_Chrll_II72 & Pa64_Chrll_1013 & $Y$ & $\mathrm{~N}$ & $\mathrm{~N}$ & Y \\
\hline 93II_Chr05_0084 & Pa64_Chr05_008I & $Y$ & $\mathrm{~N}$ & $Y$ & Y \\
\hline 93II_Chr07_0583 & Pa64_Chr07_0573 & $Y$ & $\mathrm{~N}$ & $Y$ & $Y$ \\
\hline 93II_Chrll_0059 & Pa64_Chrll_0066 & $Y$ & $\mathrm{~N}$ & $Y$ & Y \\
\hline 93II_Chr0I_0387 & Pa64_Chr0l_045I & $Y$ & $Y$ & $Y$ & Y \\
\hline 931I_Chr03_0613 & Pa64_Chr03_0602 & $Y$ & $Y$ & $Y$ & Y \\
\hline 931I_Chr03_I065 & Pa64_Chr03_1056 & $Y$ & $Y$ & $Y$ & $Y$ \\
\hline 931I_Chr04_0532 & Pa64_Chr03_0450 & $Y$ & $Y$ & $Y$ & $Y$ \\
\hline 9311_Chr06_3185 & Pa64_Chr06_280I & Y & $Y$ & $Y$ & Y \\
\hline
\end{tabular}

$\mathrm{N}$ stands for insignificant differences (less than $3 \mathrm{bp}$ in the sequence) and $\mathrm{Y}$ stands for significant differences (more than 3 bp in the sequence or motif differences).

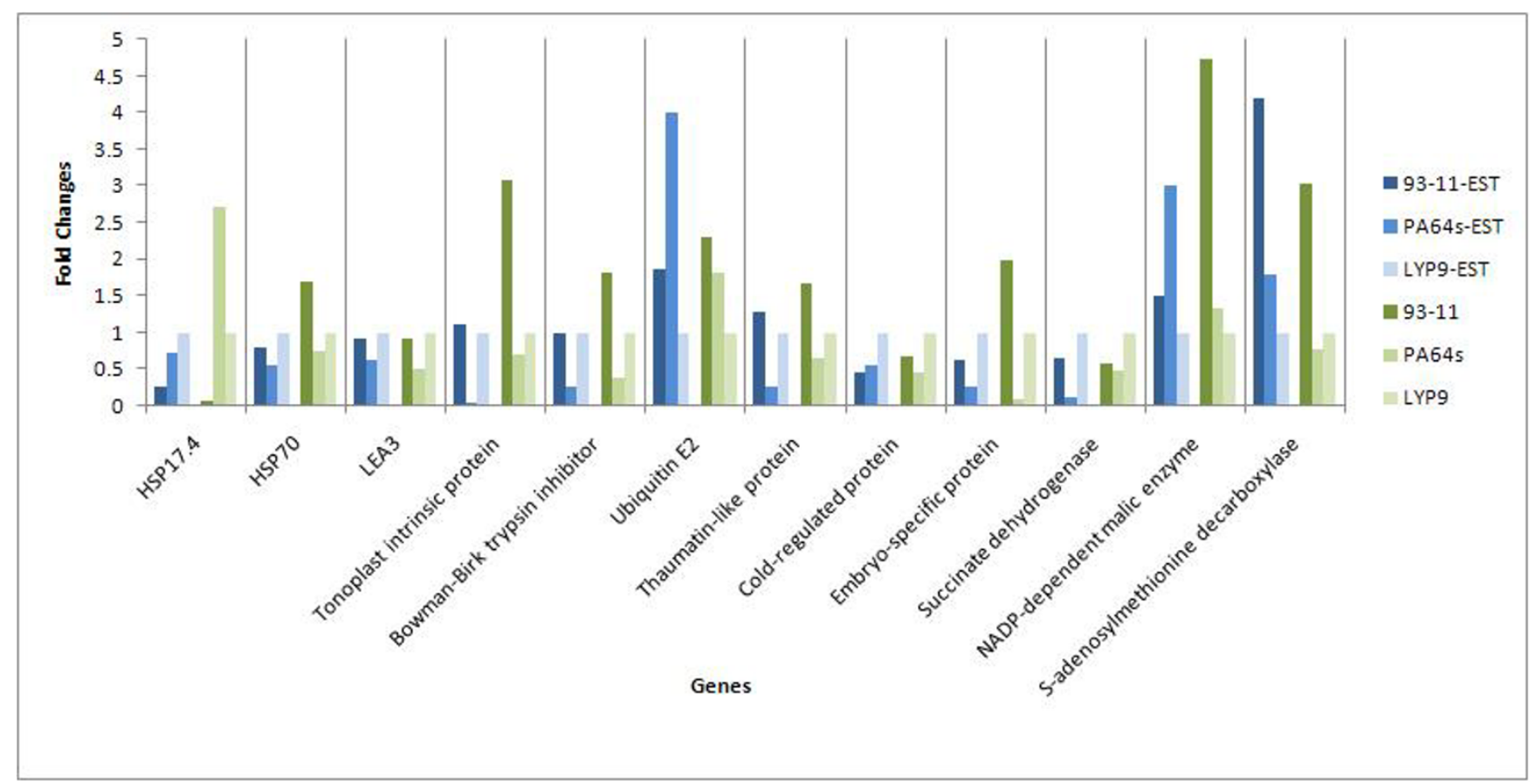

Figure 7

qRT-PCR verification. The quantitative real-time PCR results confirmed the expression variations among samples. The EST section showed the fold changes detected by cluster size. 
Table 10: Stress-associated genes

\begin{tabular}{|c|c|c|c|c|c|c|}
\hline \multicolumn{7}{|c|}{ Water-deficit Tolerance Genes } \\
\hline \multirow[t]{11}{*}{ Protection } & \multicolumn{2}{|l|}{ Name (Unigene ID) } & \multicolumn{4}{|c|}{ Expression level } \\
\hline & & & $93-11$ & PA64s & LYPQ & Total \\
\hline & WSI & WSII 8 (Unigene_0040) & 28 & 6 & 43 & 77 \\
\hline & & WSI76 (Unigene_006I) & 12 & 6 & 22 & 40 \\
\hline & Tonoplast intrinsic protein & I.I (Unigene_0034) & 50 & 2 & 45 & 97 \\
\hline & & 3.I (Unigene_0074) & 16 & 5 & 16 & 37 \\
\hline & & 1.2 (Unigene_0378) & I & 1 & 5 & 7 \\
\hline & Aquaporin PIP2.I (Unigene & & 4 & I & 6 & 11 \\
\hline & Water channel protein (Uni & & 12 & 10 & 7 & 29 \\
\hline & Bowman Birk trypsin inhibit & & 49 & 13 & 49 & III \\
\hline & Lectin (Unigene_0006) & & 106 & 53 & 97 & 256 \\
\hline \multirow[t]{5}{*}{ Degradation } & Ubiquitin E2 (Unigene_00I8 & & 41 & 88 & 22 & 151 \\
\hline & Cysteine proteinase (Uniger & & 17 & 16 & 24 & 57 \\
\hline & Aspartic proteinase oryzasir & 76) & 8 & 5 & 2 & 15 \\
\hline & Aspartic protease (Unigene & & 5 & 6 & 5 & 16 \\
\hline & Asparaginyl endopeptidase & $0083)$ & 8 & 20 & 5 & 33 \\
\hline \multicolumn{7}{|c|}{ Other Abundantly Expressed Stress-associated Genes } \\
\hline \multirow[t]{8}{*}{ Protection } & Name(Unigene ID) & & \multicolumn{4}{|c|}{ Expression level } \\
\hline & & & $93-11$ & PA64s & LYP9 & Total \\
\hline & Thaumatin-like protein (Uni & & 111 & 22 & 86 & 219 \\
\hline & Gytochrome P450 monoox & e_000I) & 1111 & 348 & 1280 & 2739 \\
\hline & NAD-/NADP-dependent ox & Jnigene_0013) & 61 & 25 & 111 & 197 \\
\hline & Heavy metal transport/deto & n (Unigene_0I07) & 4 & 9 & 14 & 27 \\
\hline & Universal stress protein (Ur & Unigene_0234) & 4 & 4 & 4 & 12 \\
\hline & & & 7 & 2 & 3 & 12 \\
\hline
\end{tabular}

Three gene families-Em, Ec, and germin proteins-have been suggested to regulate the development of rice embryo to seedling [15]. The Em and Ec proteins are encoded by conserved mRNAs stored in mature embryos but germin transcribes after growth recovery. The Em protein is involved in the process of seed desiccation, and the Ec protein is the only bona fide $\mathrm{Zn}$ metallothionein yet found in higher plants [15]; both may play important roles in ion homeostasis during embryogenesis since Em and Ec proteins are expressed as the most abundantly expressed genes in our dataset with 379 and 138 ESTs, respectively. In addition, we identified one embryogenic cell protein 40 in the high-abundance gene class as well as other development-associated proteins including embryo-specific protein, differentiation embryo protein 31 , and early embryogenesis protein in medium-abundance genes.

Pathway analysis on the mRNA repertoire provided a better glance on the basic physiology of rice mature embryos detected by pathway analysis, which showed that the embryo is well-prepared for rapid development. First, genes in the pathways of metabolism, especially the subsets of carbon, energy, and amino acid metabolisms, are stored in high abundance. Material utilization and energy production, such as glycolysis $(10.4 \%$ of carbon metabolism), TCA cycle, starch and sucrose metabolism, oxidative phosphorylation, and carbon fixation are of essence for the embryo. Second, genes participating in translation (43.4\% of GIP) and sorting (39.0\% of GIP) are in high abundance to ensure rapid translation of the reserved RNA. Third, in the category of EIP, signal transduction (58.1\% of EIP) and membrane transport (41.9\% of EIP) stood out obviously to guarantee rapid signal transduction in the recovery phase, especially the MAPK signal pathway that is important in regulating cell cycle. Fourth, the defence (endocrine and immune) system is the highly-expressed gene category in our dataset for cellular processes, which ensures hormonal regulation in development and protection.

Our detailed analyses indicated that several important pathways active in mature plant tissues are almost absent in embryos. For example, we only identified nine ESTs 
involved in photosynthesis and none involved in respiration from our dataset. Although present at low level, transcription (11.1\% of GIP) and replication/repair (6.4\% of GIP) genes are important for growth recovery as compared to the complete absence of genes in the categories of signal molecules and interaction as well as the low level of cellular process genes in our dataset. The reservoir of the reserved mRNAs in the mature embryo appeared wellprepared for basic metabolism, response to environmental stimulus, and signal transduction to ensure rapid recovery. In the contrary, secondary metabolism, cellular process, and transcription constitute a relatively small portion of the total, and most of these functions must depend upon new mRNA syntheses.

\section{Possible biological mechanisms of heterosis}

When displaying all DEGs into two-dimension space, we found a composite expression pattern that suggests multiple modes of action as they are classified into 5 patterns: over-dominance, high-parent dominance, additivity, lowparent dominance and under-dominance (Table 7). Although up- and down-regulated DEGs are almost equal in numbers (Figure 6), up-regulated genes are dominant in the most abundant gene classes including cellular, metabolic, response to stress, embryo development, enzyme regulator activity, transcription regulator activity, and transporter activity (figure 8).

\section{Strengthened stress-tolerance system in FI}

The majority of stress-tolerance genes in our dataset exhibited differential expressions among the hybrid triad, and especially showed up-regulated expressions in LYP9 (Table 6 and Table 10). In the GO term, five out of seven $(71.4 \%)$ DEGs are in the category of response to stress and up-regulated in F1. In the gene families related to stress tolerance (HSP, LEA, and RAB), 70.4\% were differentially expressed whereas $89 \%$ of them are up-regulated together with 5.5\% down-regulated and 5.5\% additivity in F1. And as shown in Table 10, among $65 \%$ of the DEGs, $77 \%$ of them are up-regulated (protective genes) as opposed to $23 \%$ down-regulated (the degradation genes). Functionally, stress-tolerance genes are classified into two groups: protective and injury-induced genes. Taking the water-deficit-tolerance related genes as examples, we know that protective genes, including chaperones (mainly LEAs and HSPs), protease inhibitors, water-deficit induced mem-

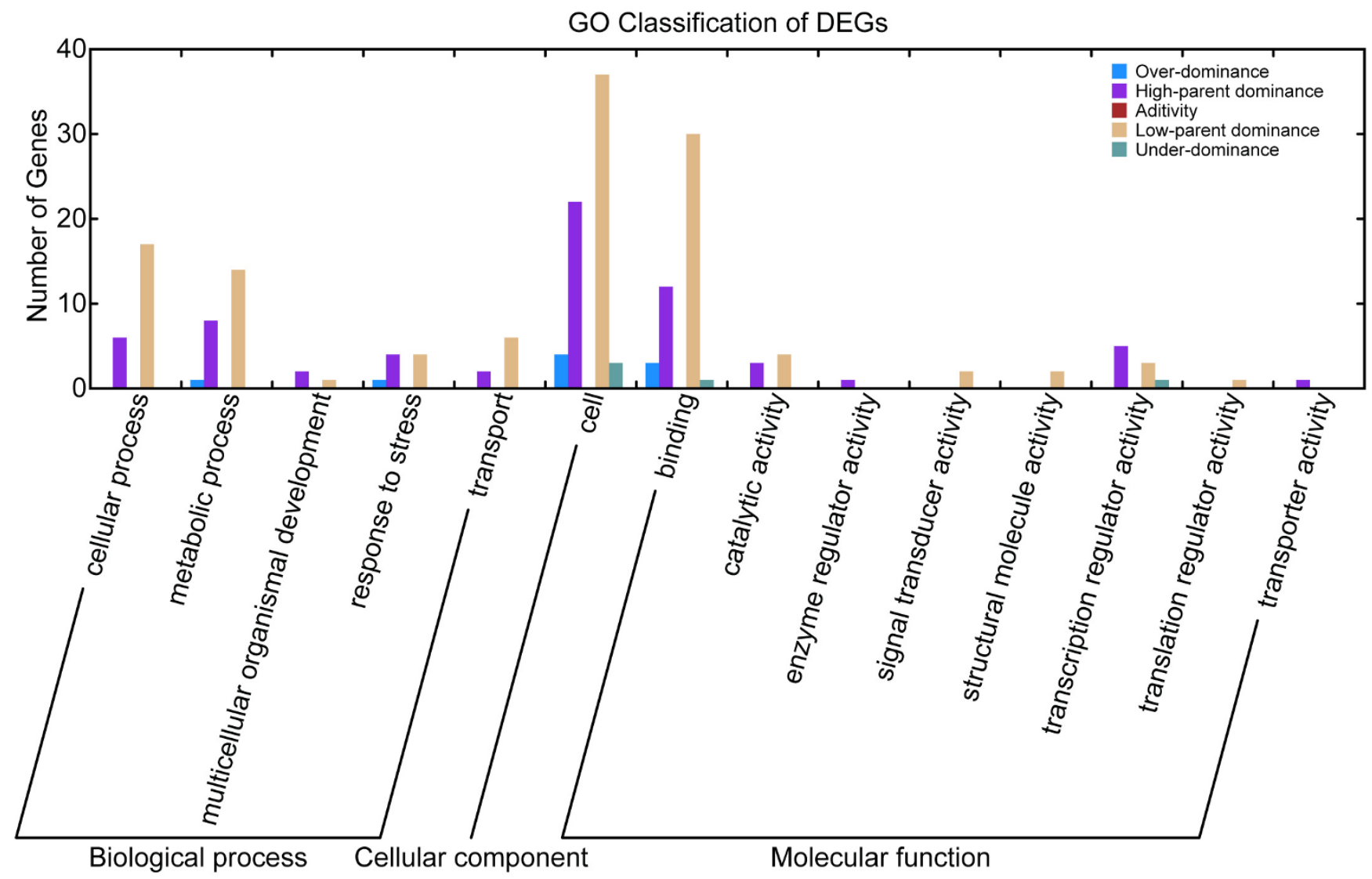

Figure 8 GO classification of DEGs. 
brane proteins, and water channel proteins, are counterparts of the protein-degrading mechanism, promoting survivability under water-deficit conditions. Injuryinduced genes include proteases and ubiquitin system, which are involved in the degradation of proteins that are denatured during cellular water loss (Figure 9). In LYP9, most of the protective genes are up-regulated as compared to the down-regulated injury-induced genes. Protective genes that participate in other stress-related functions are also up-regulated in the F1. Proteomic data also added footnotes to this notion, where the most functionally important and representative genes, including LEA3, sHSP, Bowman-Birk trypsin inhibitor, Lipoprotein and lectin, thaumatin-like protein were found as up-regulated DEGs. In addition, our SAGE data also pointed out a universal strengthening in the ability of stress-tolerance in the F1, where heavy metal transport/detoxification protein, senescence-associated protein and RAB21 protein were found up-regulated albeit in other tissues of LYP9. We validated some of these results with qRT-PCR.

\section{Better preparation for growth recovery}

DEG analysis suggested that the F1 appears better prepared for growth recovery as compared to its parental lines. First, genes participating in material and energy utilization are up-regulated and genes participating in anabolism are down-regulated (Additional file 8: Summary of DEGs in pathway analysis). In general, the up-regulated DEGs were found in carbon metabolism with an exception of the oxidative phosphorylation of a NADP-dependent malic enzyme in energy metabolism. In contrast, genes in carbon, nitrogen fixation, and nucleotide metabolism are mostly down-regulated. In amino acid metabolism, we identified genes both up-regulated and downregulated, but all genes related to amino acid biosynthesis are down-regulated. This indicated that LYP9 up-regulate material and energy generation and utilization to ensure its embryo to be active immediately when being rehydrated as material anabolism is normally down-regulated. Second, in the category of GIP, genes involved in protein folding and associated processing are up-regulated and genes of ubiquitin mediated proteolysis and proteasome are down-regulated. Genes involved in tran-

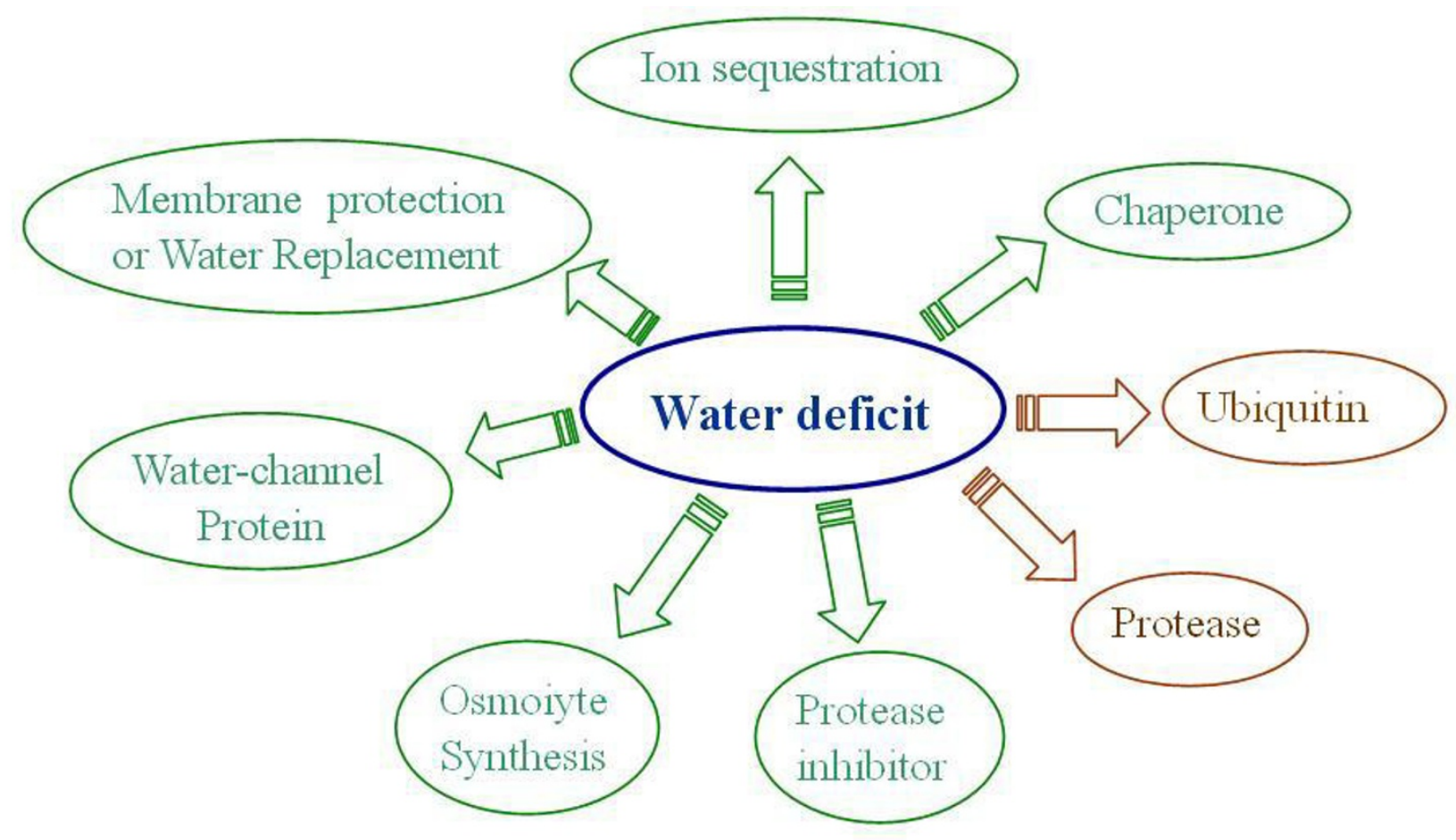

Protection associated genes

Injury induced genes

\section{Figure 9}

DEGs in water-deficit tolerance system. Note that genes playing protective roles are all up-regulated whereas those injury-induced are down-regulated. 
scription are down-regulated but those involved in translation are not obviously biased, where both down- and up-regulated genes were found. Third, in EIP, genes in membrane transport and MAPK signal transduction are up-regulated but calcium signalling pathway and phosphatidylinositol signalling systems are down-regulated; the result suggested that MAPK signalling pathway may be the major signal pathway triggered as development starts after re-hydration. Finally, DEGs involved in cellular process but not in the response (immune) system appeared down-regulated. Some of these genes and their trends of expressions were found consistent in our SAGE and proteomic data. For example, the up-regulation of an important enolase was confirmed in the protein data. And the up-regulation of succinate dehydrogenase was validated based on the qRT-PCR test. Another instance is $\beta$-amylase that is a key enzyme for starch hydrolysis, which is not only found up-regulated in the embryo but also in the leaf and panicle of LYPY.

\section{Complementation and over-dominance}

We found that embryo-characteristic genes among DEGs are primarily up-regulated and showed high-parent dominance $(79.2 \%)$ with the addition of over-dominance $(20.8 \%)$. The high-parent dominance genes often occur in gene families. For example, in the HSP family, the expression of LMW HSPs has the tendency of PA64s, higher than that of 93-11, or expressed in an over-dominance fashion. In contrast, the expression level of classical HSPs resembles that of 93-11, which is higher than that of PA64s, exhibiting over-dominance. In the LEA and RAB family, as well as other stress-tolerance genes, all the DEGs show high-parent dominance with expression levels similar to 93-11. More than two third of the DEGs differ between PA64s and LYP9 (72.7\%), whereas less than one third show differences between 93-11 and LYP9 (27.3\%). The result is consistent with the fact that LYP9 is more like 9311 than PA64s in their phenotypic appearances (Lihuang Zhu and Qingzhong Xue, personal communications). Therefore, it appears that the hybrid vigour of LYP9 may be contributed by a composite of beneficial alleles from both parents, complementing the relatively weaker alleles in a global fashion, and over-dominance is an essential strategy for stress tolerance and metabolism.

\section{Strengthening tissue-characteristic functions may contribute to heterosis}

Analyses on DEGs have indicated that the mode of action for heterosis is most likely multi-fold and at multiple levels. If we have to point out a single mode of action, we believe that the strengthening of embryo-characteristic genes in LYP9 is most noticeable. For instance, the F1, $L Y P 9$, is predicted to have a significantly better stress-tolerance system than its parental cultivars, supported by upregulated protective genes and down-regulated injury- induced genes. In addition, LYP9 keeps strong responses to environmental signals and potentially high-level activities after growth recovery. Therefore, when seeds rehydrate, the F1 embryo may perform better than its parental lines. This phenomenon can be cautiously generalized based on similar analyses among other tissues of the same hybrid line. For example, in leaf, it was detected that upregulated genes in hybrid are dominated by DEGs related to enhancing carbon assimilation whereas those related to decomposition of carbohydrates are down-regulated [10], resulting in an increased efficiency of photosynthesis or energy production.

\section{Conclusion}

We reported a comparative transcriptomic analysis based on ESTs acquired from mature embryos of a rice hybrid and its parental lines. Our study not only provided an initial characteristic description of expression profiles of rice embryos but also pointed out the enrichment of genes involved in stress-tolerance and preparation-for-recover (both development and physical activity). We also identified 191 DEGs between F1 and its parental lines. Functional analyses revealed clues for rice heterosis, such as multiple modes of actions and strengthening of embryocharacteristic function. Genes involved in stress-tolerance and preparation-for-recovery may play key roles for rice heterosis in mature embryos.

\section{Methods}

\section{Plant materials and cDNA libraries}

A fresh-harvest of mature seeds for LYP9 (F1 embryo harvested from the maternal plant,PA64s) and its parental lines 93-11 and PA64s were collected and stored at room temperature. The seeds were provided by the Chinese National R\&D Center on Hybrid Rice, Changsha, China, and the plants were grown in its experimental rice paddies $\left(28^{\circ} 30 \mathrm{~N}, 113^{\circ} 40 \mathrm{E}\right.$, and altitude $\left.40 \mathrm{~m}\right)$. Mature rice embryos were separated manually from the seeds by using scalpels and stored in liquid nitrogen immediately until RNA isolation.

We constructed cDNA libraries for each rice cultivar, using a combined priming method, i.e. used both oligo-dT- and random-priming to avoid potential cloning biases. Briefly, total RNA was extracted from dissected embryos with Trizol (Invitrogen), and mRNAs was purified with Oligotex mRNA Midi Kit (QIAGEN). Reverse transcription was carried out separately by using oligo-dT (with Xhol-linker sequence) and random primers in equal amount. For oligo-dT-primed cDNA, the second strand was synthesized and linked to an EcoRI-linker. After XhoI digestion, insert DNA was fractioned (1-2 Kb) with QIAquick Gel Extraction Kit (QIAGEN) and cloned into a directional pBluescript ${ }^{\circledast}$ II XR vector (Stratagene). For ran- 
dom-primed cDNA library, fractioned cDNA was cloned into a pUC18 vector.

\section{EST assembly and annotation}

We picked approximately 7,000 clones from each oligoprimed library and over 3,000 clones from each randomprimed library for single-pass unidirectional sequencing from the 5'-end (MegaBase 1000 sequencers). We used Phred $[20,21]$ for base-calling, rejected low-quality sequences according to default settings, and removed lowquality sequences shorter than $100 \mathrm{bp}$. Cross_match program was used to trim vector sequences. We also removed ESTs that do not have significant similarities [22] with the collection of Oryza sativa Gene Index database (release_17, it contains the largest assembly of publicly available rice ESTs) and the reference genomes including predicted genes from 93-11 [11]. We used Phrap (minmatch 50 and minscore 100) and Consed [23] to build consensus sequences and to view the assemblies. We further aligned all unigenes to NCBI nr-databases and clustered unigenes with the same gi-numbers to form nonredundant unigenes after manual inspections. In addition, we calculated the expression abundance for all unique sequences or unigenes based on ESTs.

We first annotated our data based on sequence similarity searches, using blast-based tools [22] (tblastx) against several databases, including NCBI non-redundant protein database, two public rice genome annotation databases (BGI rice genome annotations and TIGR rice pseudomolecule release 5), two major rice EST assembly databases (NCBI UNIGENE release 62 and TIGR OGI release 17) with an e-value cutoff of $1 e-5$. We then assigned functional categories for the unigenes according to GO functional classification using a web-based tool WEGO [24]http://wego.genomics.org.cn/cgi-bin/wego/index.pl and biological processes/pathways using the online KEGG Automatic Annotation Service http://www.genome.jp/ kegg/kaas/ with the BBH option checked.

\section{Defining specifically-expressed and differentially- expressed genes}

We selected eight representative libraries from the NCBI Digital-Differential-Display (DDD) database for a comparative analysis (Table 5). We measured gene expression abundance as transcript per million (TPM) and sorted the expression abundance and annotation for every unigene among different tissues. Then do the hierarchical clustering analyses using default parameters. The parallel analysis with SAGE data was based on rice genome annotations with similar parameters. The GO classification analysis on both predicted genes from the parental (93-11 and PA64s) genomes and the mature embryo were performed and categories that has a larger percent of genes in embryo than genome were supposed to be enriched than genome general transcriptome.

DEGs among the three cultivars were determined by using IDEG6 $[25,26]$. The cutoff value was set to $p<=0.05$ for general Chi-squared test. All DEGs were classified into twelve offspring-parent distribution modes according to their expression patterns. Fold changes were detected based on the equation LYP9/[(93-11+PA64s)/2]. The radius at which a gene is plotted represents fold change and the position where each spot laid depends on the expression relationship among three libraries.

\section{Quantitative Real-time PCR validation}

We selected 12 functionally important and representative DEGs for validation using quantitative real-time PCR (qRT-PCR). Total RNA was digested with RNase-free DNase I to remove DNA contaminations and reverse-transcribed with a mixed primers (oligo-dT primer: random primer in a molar ratio of $=7: 3$ ). Gene-specific primers were designed for each DEG. and the rice $\beta$-actin gene was used as a control. qRT-PCR was performed by using a Quant SYBR Green PCR kit (Tiangen, china). The results were based on the average of three parallel experiments and analysed with the Opticon Monitor software. Melting curves and CT (cycle threshold) values for DEGs were used to measure expression levels. We calculated standard deviations for parallel samples, set cut-off standard to one, and quantified gene expression based on normalization with the control and self-normalization in parallel samples.

\section{Authors' contributions}

XG constructed the cDNA libraries, prepared clones for sequencing, performed data processing, functional analyses of ESTs, and qRT-PCR as well as drafted the manuscript. WC provided guidance in data processing and analysis. WW and SS helped in organizing and revising the manuscript. JY and SH supervised the study and revised the manuscript. All authors read and approved the final manuscript.

\section{Additional material}

\section{Additional file 1}

Summary of unigenes. We summarized expression abundance and distribution in three libraries of all unigenes as well as their best hits in NCBI nr database (NA: no hit).

Click here for file

[http://www.biomedcentral.com/content/supplementary/14712229-8-114-S1.xls] 


\section{Additional file 2}

A full list of unigene sequences. We listed all unigene sequences assembled from ESTs.

Click here for file

[http://www.biomedcentral.com/content/supplementary/14712229-8-114-S2.txt]

\section{Additional file 3}

Hierarchical clustering of medium-abundance genes. We clustered medium-abundance genes to show that its majority are also enriched in mature rice embryo as compared to other representative tissues chosen from NCBI DDD database (A) and the SAGE data from the same hybrid line ( $B$ and $C)$.

Click here for file

[http://www.biomedcentral.com/content/supplementary/14712229-8-114-S3.tiff]

\section{Additional file 4}

DEGs and their expression patterns. We provided the unigene ID, expression pattern, and P-value for each DEG. N, P and L stand for 9311, PA64s and LYP9 respectively. A hyphen denotes the absence of expression in EST data.

Click here for file

[http://www.biomedcentral.com/content/supplementary/14712229-8-114-S4.xls

\section{Additional file 5}

DEGs shared with SAGE data. We marked the expression pattern of DEGs in other tissues with up and down arrows. A hyphen denotes the absence of differential expression.

Click here for file

[http://www.biomedcentral.com/content/supplementary/14712229-8-114-S5.xls]

\section{Additional file 6}

Primers for $q R T-P C R$. We provided primer sequences used in $q R T-P C R$. Click here for file [http://www.biomedcentral.com/content/supplementary/14712229-8-114-S6.xls]

\section{Additional file 7}

GO classification of universally-expressed genes among embryo and other tissues from SAGE data. We classified genes that universally expressed at similar expression level among embryo and other tissues from SAGE data based on basic categories of Gene Ontology.

Click here for file

[http://www.biomedcentral.com/content/supplementary/14712229-8-114-S7.pdf]

\section{Additional file 8}

Summary of DEGs in pathway analysis. We showed unigene ID, expression pattern, and associated pathway for all DEGs.

Click here for file

[http://www.biomedcentral.com/content/supplementary/14712229-8-114-S8.xls]

\section{Acknowledgements}

Matured seeds were provided by Chinese National Hybrid Rice R\&D

Center. The work received financial support from Chinese Academy of Sci- ence (KSCXI-SW-03), the Ministry of Science and Technology (2005AA235 I I0), and the National Basic Research Program of China (2006CB9 10404) awarded to JY. This project is also supported by a grant (3022 1004) from the National Natural Science Foundation of China.

\section{References}

I. Lippman ZB, Zamir D: Heterosis: revisiting the magic. Trends Genet 2007, 23(2):60-66

2. Crow JF: Alternative Hypotheses of Hybrid Vigor. Genetics 1948, 33(5):477-487.

3. Shull G: The Composition of a Field of Maize. Am Breed Assn Rep |908, 4:296-30|.

4. Xiao J, Li J, Yuan L, Tanksley SD: Dominance Is the Major Genetic Basis of Heterosis in Rice as Revealed by QTL Analysis Using Molecular Markers. Genetics 1995, I 40(2):745-754.

5. Luo LJ, Li ZK, Mei HW, Shu QY, Tabien R, Zhong DB, Ying CS, Stansel JW, Khush GS, Paterson AH: Overdominant epistatic loci are the primary genetic basis of inbreeding depression and heterosis in rice. II. Grain yield components. Genetics 200I, I 58(4): |755-|77|.

6. Li ZK, Luo LJ, Mei HW, Wang DL, Shu QY, Tabien R, Zhong DB, Ying CS, Stansel JW, Khush GS, et al.: Overdominant epistatic loci are the primary genetic basis of inbreeding depression and heterosis in rice. I. Biomass and grain yield. Genetics 200I, I58(4): 1737-1753.

7. Milborrow B: A biochemical mechanism for hybrid vigour. Exp Bot 1998, 49(324): 1063-107|.

8. Shibata K: [Various methods for gene expression analysis]. Tanpakushitsu kakusan koso 2004, 49(I7 Suppl):2694-2700.

9. Swanson-Wagner RA, jia Y, DeCook R, Borsuk LA, Nettleton D, Schnable PS: All possible modes of gene action are observed in a global comparison of gene expression in a maize FI hybrid and its inbred parents. Proceedings of the National Academy of Sciences of the United States of America 2006, 103(18):6805-68I0.

10. Song S, Qu H, Chen C, Hu S, Yu J: Differential gene expression in an elite hybrid rice cultivar (Oryza sativa, $L$ ) and its parental lines based on SAGE data. BMC Plant Biol 2007, 7(I):49.

II. Yu J, Hu S, Wang J, Wong GK, Li S, Liu B, Deng Y, Dai L, Zhou Y, Zhang $X$, et al:: $\mathbf{A}$ draft sequence of the rice genome (Oryza sativa L. ssp. indica). Science (New York, NY) 2002, 296(5565):79-92.

12. Goff SA, Ricke D, Lan TH, Presting G, Wang R, Dunn M, Glazebrook J, Sessions A, Oeller $P, V a r m a ~ H$, et al.: A draft sequence of the rice genome (Oryza sativa L. ssp. japonica). Science (New York, NY) 2002, 296(5565):92-100.

13. Wang $Q$, Zhang QD, Jiang GM, Lu CM, Kuang TY, Wu S, Li CQ, Jiao DM: Photosynthetic Characteristics of Two Superhigh-yield Hybrid Rice. Acta Botanica Sinica 2000, 42(I 2): I 285-I 288.

14. Bao J, Lee S, Chen C, Zhang X, Zhang Y, Liu S, Clark T, Wang J, Cao $M$, Yang $H$, et al.: Serial analysis of gene expression study of a hybrid rice strain (LYP9) and its parental cultivars. Plant physiology 2005, I 38(3): | $216-123 \mid$.

15. Lane BG: Cellular desiccation and hydration: developmentally regulated proteins, and the maturation and germination of seed embryos. FASEB J I991, 5( I 4):2893-2901.

16. Meyer S, Pospisil H, Scholten S: Heterosis associated gene expression in maize embryos 6 days after fertilization exhibits additive, dominant and overdominant pattern. Plant Mol Biol 2007, 63(3):38I-39I.

17. Bray EA: Molecular Responses to Water Deficit. Plant Physiol 1993, 103(4): 1035-1040.

18. Ingram J, Bartels D: The Molecular Basis Of Dehydration Tolerance In Plants. Annu Rev Plant Physiol Plant Mol Biol 1996, 47:377-403.

19. Song S, Qu H, Chen C, Hu S, Yu J: Differential gene expression in an elite hybrid rice cultivar (Oryza sativa, $L$ ) and its parental lines based on SAGE data. BMC Plant Biol 2007, 7:49.

20. Ewing $B$, Green P: Base-calling of automated sequencer traces using phred. II. Error probabilities. Genome Res 1998, 8(3): $186-194$

21. Ewing B, Hillier L, Wendl MC, Green P: Base-calling of automated sequencer traces using phred. I. Accuracy assessment. Genome Res 1998, 8(3): 175-185.

22. Altschul SF, Madden TL, Schaffer AA, Zhang J, Zhang Z, Miller W, Lipman D!: Gapped BLAST and PSI-BLAST: a new generation of 
protein database search programs. Nucleic Acids Res 1997, 25(I7):3389-3402.

23. Gordon D, Abajian C, Green P: Consed: a graphical tool for sequence finishing. Genome Res 1998, 8(3): 195-202.

24. Ye J, Fang L, Zheng H, Zhang Y, Chen J, Zhang Z, Wang J, Li S, Li R, Bolund L, et al.: WEGO: a web tool for plotting GO annotations. Nucleic acids research 2006:W293-297.

25. Romualdi C, Bortoluzzi S, D'Alessi F, Danieli GA: IDEG6: a web tool for detection of differentially expressed genes in multiple tag sampling experiments. Physiol Genomics 2003, 12(2):159-162.

26. Romualdi C, Bortoluzzi S, Danieli GA: Detecting differentially expressed genes in multiple tag sampling experiments: comparative evaluation of statistical tests. Hum Mol Genet 200I, I0(19):2|33-2|4I.

Publish with Bio Med Central and every scientist can read your work free of charge

"BioMed Central will be the most significant development for disseminating the results of biomedical research in our lifetime. "

Sir Paul Nurse, Cancer Research UK

Your research papers will be:

- available free of charge to the entire biomedical community

- peer reviewed and published immediately upon acceptance

- cited in PubMed and archived on PubMed Central

- yours - you keep the copyright

Submit your manuscript here:

http://www.biomedcentral.com/info/publishing_adv.asp
BioMedcentral 